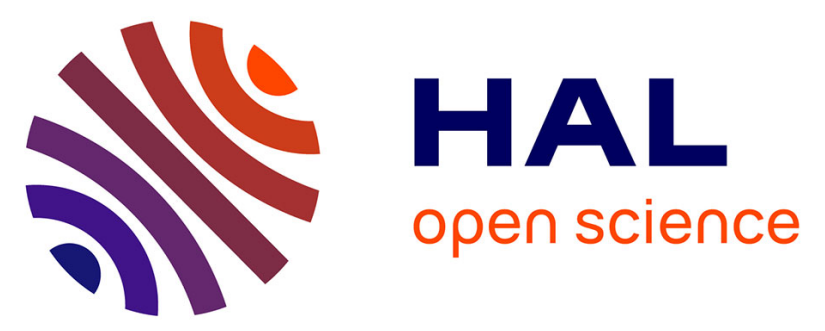

\title{
A variational constitutive update algorithm for a set of isotropic hyperelasticviscoplastic models
}

\author{
Eduardo Fancello, Jakson M Vassoler, Laurent Stainier
}

\section{To cite this version:}

Eduardo Fancello, Jakson M Vassoler, Laurent Stainier. A variational constitutive update algorithm for a set of isotropic hyperelasticviscoplastic models. Computer Methods in Applied Mechanics and Engineering, 2008, 197 (49-50), pp.4132-4148. 10.1016/j.cma.2008.04.014 . hal-01004964

\section{HAL Id: hal-01004964 \\ https://hal.science/hal-01004964}

Submitted on 18 Nov 2021

HAL is a multi-disciplinary open access archive for the deposit and dissemination of scientific research documents, whether they are published or not. The documents may come from teaching and research institutions in France or abroad, or from public or private research centers.
L'archive ouverte pluridisciplinaire HAL, est destinée au dépôt et à la diffusion de documents scientifiques de niveau recherche, publiés ou non, émanant des établissements d'enseignement et de recherche français ou étrangers, des laboratoires publics ou privés.

\section{(ㄷ)(1) $\$$}

Distributed under a Creative Commons Attribution - NonCommerciall 4.0 International 


\title{
A variational constitutive update algorithm for a set of isotropic hyperelastic-viscoplastic material models
}

\author{
Eduardo Fancello ${ }^{\mathrm{a}, *}$, Jakson M. Vassoler ${ }^{\mathrm{a}, 1}$, Laurent Stainier ${ }^{\mathrm{b}, 2}$ \\ a Departmento de Engenharia Mecânica, Universidade Federal de Santa Catarina, Campus Universitário, Trindade, Florianópolis, SC, Brazil \\ ${ }^{\mathrm{b}}$ Département Aérospatiale and Mécanique, Université de Liège, B-4000 Liège, Belgium
}

\begin{abstract}
In three-dimensional formulations of plasticity, the rate of plastic deformation is usually decomposed in direction and amplitude. In the most simple cases of von Mises type flows, this allows to assume a known radial flow direction and computations are reduced to the determination of the amplitude of the plastif-ication. In the context of a variational formulation of finite plasticity, it is verified that this decomposition between constitutive and kinematic aspects is accomplished by the choice of a quadratic elastic potential (Hencky model). The aim of this paper is to show that a wide set of simple hyperelastic-plastic isotropic models not restricted to quadratic elastic behavior can be constructed by relaxing the classical decompo-sition amplitude/ direction by the sum of spectral quantities. In addition, this approach allows for a uni-fied and efficient numerical implementation in which a particular hyperelastic-viscoplastic behavior is obtained by a specific choice of the elastic, plastic and dissipative (pseudo)potentials.
\end{abstract}

\section{Introduction}

Variational principles for dissipative mechanical systems, such as in presence of elasto-viscoplastic behavior, have been known for a while, but have received renewed attention in recent years. These principles can be written in a continuous or in an incremental framework (a non-exhaustive list would among others include [1-3], and references therein). In particular, a variational formulation of constitutive models for standard generalized materials [4], including irreversible, dissipative, and possibly rate-dependent behaviors, was proposed in [5,6], initially in an isothermal context, and later extended to a fully coupled thermo-mechanical context in [7]. One of the most relevant aspects of variational approaches is that they provide appropriate mathematical basis for error estimation and mesh adaption [6,8]. This framework has also been used for developing models of non-cohesive granular media [9], porous plasticity [10], and nonlinear finite viscoelasticity [11]. In this latter work in particular, a spectral decomposition of elastic/ inelastic strain/strain-rate quantities was proposed in order to facilitate the inclusion of arbitrary elastic and inelastic (isotropic) potentials within the same formalism.

\footnotetext{
* Corresponding author. Tel.: +55 4837219899 .

E-mail addresses: fancello@grante.ufsc.br (E. Fancello), jaksonmv@hotmail.com (J.M. Vassoler), L.Stainier@ulg.ac.be (L. Stainier).

1 Present address: Dipartimento di Meccanica, Politecnico di Torino - Corso Duca degli Abruzzi, 2410129 Torino, Italy.

2 Research Associate at the Belgian F.R.S.-FNRS.
}

In elasto-plasticity and elasto-viscoplasticity, the rate tensor of plastic deformation (or its incremental equivalent), is usually decomposed in direction, related to the gradient of a yield potential, and magnitude. In the case of von Mises (J2) type flows and small strain ranges, this decomposition provides a complete separation between kinematic and constitutive aspects which reduces the problem to the determination of the plastification in a radial known direction. This approach is known as the radial return algorithm [12,3]. Analogous results are obtained with classical hyperelastic-based models by using appropriate logarithmic strain measures and exponential integration algorithm [13-16]. In the variational approach, both internal quantities are determined by a local minimization process. Moreover, it is shown in [5] that the kinematic and constitutive decoupling is, once again, achieved by the choice of logarithmic strain measures, exponential integration algorithm and quadratic elastic potential (Hencky model).

The Hencky model is usually appropriate for the representation of the elastic behavior of metals in which the elastic strains are small compared the plastic ones. However, this is not the case for many polymers, whose behavior is frequently classified as viscoelastic or viscoplastic depending on the regime of strains and strain rates to which they are submitted.

The aim of this paper is to show that, embedded in the variational framework, decoupling properties similar to those mentioned above may be extended to a wide set of simple hyperelastic-viscoplastic isotropic models not restricted to quadratic elastic behavior. This is performed by relaxing the classical decomposition 
amplitude/direction in a similar way as done in [11], by using spectral quantities.

This approach was adopted to potentially allow for a natural combination of viscoelastic and viscoplastic dissipative mechanisms appropriate for a group of thermoplastic polymers, which is the longer-term motivation of this work.

The paper is organized as follows: Section 2 briefly presents the variational approach for irreversible constitutive problems. The application of this approach to elasto-viscoplastic materials is stated in Section 3 where important results of this work are shown. Section 4 is dedicated to discuss how the characterization of the yield limit comes out as a natural consequence of the present approach. Section 5 shows two particular material models within the present context while Section 6 presents some numerical examples showing the behavior of the constitutive problem itself and its performance in a general finite element code. Final remarks are shown in Section 7.

\section{Incremental formulation for inelastic constitutive behavior}

The main assumption in hyperelasticity is the existence of a potential function $\Psi$ which depends on the value of strains only and whose derivative provides the state of stress at a material point, i.e.,

$\mathbf{P}=\frac{\partial \Psi(\mathbf{F})}{\partial \mathbf{F}}=2 \mathbf{F} \frac{\partial \Psi(\mathbf{C})}{\partial \mathbf{C}}$.

In (1) $\mathbf{F}=\nabla_{0} \mathbf{X}$ denotes the deformation gradient, $\mathbf{C}=\mathbf{F}^{\mathrm{T}} \mathbf{F}$ is the right Cauchy-Green tensor and $\mathbf{P}$ the first Piola Kirchhoff stress tensor. Assuming the satisfaction of compatibility and constitutive equations, the equilibrium problem may be defined by the minimization of the potential energy

$$
\begin{aligned}
& \min _{\mathbf{x} \in K} \mathscr{H}(\mathbf{x}) \\
& \mathscr{H}(\mathbf{x})=\int_{\Omega_{0}} \Psi(\mathbf{F}(\mathbf{x})) \mathrm{d} \Omega_{0}-\left[\int_{\Omega_{0}} \mathbf{b}_{0} \cdot \mathbf{x} \mathrm{d} \Omega_{0}+\int_{\Gamma_{0}} \mathbf{f}_{0} \cdot \mathbf{x} \mathrm{d} \Gamma_{0}\right],
\end{aligned}
$$

where $K$ is the set of admissible deformations.

On the other hand, the state of stress of an inelastic path-dependent dissipative solid cannot be obtained just from the value of final strains and it is not any longer possible to define a potential function with the property (1). The history of the process is usually described incrementally with the aid of internal (dissipative) variables. However it is shown in $[5,6]$ that a wide set of dissipative materials can be modeled by the aid of pseudo-potentials that behave like hyperelastic (in the sense that they satisfy (1)) within the interval of a load increment, i.e.,

$\mathbf{P}_{n+1}=\frac{\partial \Psi\left(\mathbf{F}_{n+1} ; \mathscr{E}_{n}\right)}{\partial \mathbf{F}_{n+1}}=2 \mathbf{F}_{n+1} \frac{\partial \Psi\left(\mathbf{C}_{n+1} ; \mathscr{E}_{n}\right)}{\partial \mathbf{C}_{n+1}}$,

where $\mathscr{E}$ denotes a set of external and internal variables:

$\mathscr{E}=\left\{\mathbf{F}, \mathbf{F}^{i}, \mathbf{Q}\right\} \quad \mathbf{F}=\mathbf{F}^{e} \mathbf{F}^{i}$.

The tensors $\mathbf{F}^{e}$ and $\mathbf{F}^{i}$ are the elastic and inelastic parts of the deformation gradient while the quantity $\mathbf{Q}$ contains all the remaining internal variables used to describe the process. The subindices $n$ and $n+1$ indicate the beginning and end of the load increment and it is assumed that all quantities at time $n$ are known.

For a quite general set of inelastic problems the potential $\Psi\left(\mathbf{F}_{n+1} ; \mathscr{E}_{n}\right)$ takes the form (see $[5,6]$ for a detailed construction):

$$
\begin{aligned}
& \Psi\left(\mathbf{F}_{n+1} ; \mathscr{E}_{n}\right)=\min _{\substack{\mathbf{F}_{n+1}^{i} \\
\mathbf{Q}_{n+1}}}\left\{W\left(\mathscr{E}_{n+1}\right)-W\left(\mathscr{E}_{n}\right)+\Delta t \psi\left(\stackrel{\circ}{\mathbf{F}}^{i}, \stackrel{\circ}{\mathbf{Q}} ; \mathscr{E}_{n}\right)\right\}, \\
& W(\mathscr{E})=U(\mathbf{F})+\varphi^{e}\left(\mathbf{F F}^{i-1}\right)+\varphi^{i}\left(\mathbf{F}^{i}, \mathbf{Q}\right),
\end{aligned}
$$

where $\stackrel{\circ}{\mathbf{F}}^{i}\left(\mathbf{F}_{n+1}^{i}, \mathscr{E}_{n}\right)$ and $\mathbf{Q}\left(\mathbf{Q}_{n+1}, \mathscr{E}_{n}\right)$ are suitable incremental approximations of the rate variables $\dot{\mathbf{F}}, \dot{\mathbf{F}}^{i}$ and $\dot{\mathbf{Q}}$ respectively. The free en- ergy potential $W$ and dissipation pseudo-potential $\psi$ inside (5) may take different expressions depending on the particular model needed. The expression of $W(\mathscr{E})$ in (6) assumes that the free energy can be additively decomposed in potentials $U, \varphi^{e}$ and $\varphi$ depending on $\mathbf{F}, \mathbf{F}^{i}$ and $\mathbf{Q}$ respectively. Their specific expressions and dependencies are related to the characteristics of each material model. In Section 3 these potentials will be particularized for a group of hyperelastic-viscoplastic models. The minimization in (5) with respect to the internal variables $\mathbf{F}_{n+1}^{i}$ and $\mathbf{Q}_{n+1}$ provides an evolution path of these variables within the time step and eliminates them from the potential $\Psi$ enforcing it to be dependent only on the deformation gradient $\mathbf{F}_{n+1}$.

\section{A set of hyperelastic-viscoplastic isotropic models}

\subsection{Definitions and hypotheses}

In this section, we focus on elastic-viscoplastic models. ${ }^{3}$ In order to introduce some useful notation, consider the classic multiplicative decomposition of $\mathbf{F}$ in isochoric and volumetric contributions:

$J=\operatorname{det} \mathbf{F}, \quad \widehat{\mathbf{F}}=\frac{1}{J^{1 / 3}} \mathbf{F}$.

In addition, the isochoric portion $\widehat{\mathbf{F}}$ allows for a multiplicative decomposition in elastic and plastic parts, such that

$\widehat{\mathbf{F}}=\widehat{\mathbf{F}}^{e} \mathbf{F}^{p} \Rightarrow \widehat{\mathbf{F}}^{e}=\widehat{\mathbf{F}} \mathbf{F}^{p-1}$

$\widehat{\mathbf{C}}^{e}=\widehat{\mathbf{F}}^{e^{\mathrm{T}}} \widehat{\mathbf{F}}^{e}=\sum_{i=1}^{3} c_{i}^{e} \mathbf{E}_{i}^{e}, \quad \operatorname{det} \mathbf{F}^{p}=1$,

where $\widehat{\mathbf{C}}^{e}$ is the isochoric elastic right Cauchy-Green tensor that is decomposed in spectral quantities: eigenvalues $c_{i}^{e}$ and eigenprojections $\mathbf{E}_{i}^{e}$. The rate of plastic deformation (or plastic stretching) $\mathbf{D}^{p}$ is defined as

$\mathbf{D}^{p}=\operatorname{sym}\left(\mathbf{L}^{p}\right)=\mathbf{L}^{p}=\dot{\mathbf{F}}^{p} \mathbf{F}^{p-1}$

where $\mathbf{L}^{p}$ is assumed to be symmetric, which means that no plastic spin $\mathbf{W}^{p}=\left(\mathbf{L}^{p}-\mathbf{L}^{p^{\mathrm{T}}}\right) / 2$ is considered. If a von Mises flow type is assumed, the tensor $\mathbf{D}^{p}$ may be decomposed as follows:

$\mathbf{D}^{p}=\dot{q} \mathbf{M}$,

$\dot{q} \in \mathbb{R}^{+}$,

$\mathbf{M} \in K_{M}=\left\{\mathbf{N} \in \operatorname{Sym}: \mathbf{N} \cdot \mathbf{N}=\frac{3}{2}, \quad \mathbf{N} \cdot \mathbf{I}=0\right\}$,

where the non-negative scalar $\dot{q}$ accounts for the amplitude of $\mathbf{D}^{p}$ while the normalized traceless tensor $\mathbf{M}$ provides the direction of $\mathbf{D}^{p}$. It is shown in [5] that, when this separation is combined with logarithmic strains and quadratic hyperelastic (Hencky type) potentials, a complete separation of kinematic aspects (provided by $\mathbf{M}$ ) and constitutive aspects (provided by $\dot{q}$ ) is reached and constitutive expressions similar to those of infinitesimal plasticity theory are obtained. This result is also verified in more classical hyperelasticplastic and viscoplastic approaches [16]. In order to extend these facilities to more general hyperelastic laws other than Hencky, a spectral decomposition of $\mathbf{D}^{p}$ is used here, following the ideas proposed in [11]:

$\mathbf{D}^{p}=\mathbf{M}=\dot{q} \sum_{i=1}^{3} q_{i} \mathbf{M}_{i}$

$\dot{q} \in \mathbb{R}^{+}, \quad q_{i} \in K_{Q}=\left\{p_{i} \in \mathbb{R}: \sum_{i=1}^{3} p_{i}=0 ; \sum_{i=1}^{3} p_{i}^{2}=3 / 2\right\}$,

$\mathbf{M}_{i} \in K_{M}=\left\{\mathbf{N}_{i} \in \operatorname{Sym}: \mathbf{N}_{i} \cdot \mathbf{N}_{i}=1, \quad \mathbf{N}_{i} \cdot \mathbf{N}_{j}=0, \quad i \neq j\right\}$.

\footnotetext{
${ }^{3}$ Just for facility of notation we change the superscript $i$ (inelastic) for $p$ (plastic).
} 
The set $K_{Q}$ enforces the traceless form of $\mathbf{M}$ with fixed norm, while the set $K_{M}$ accounts for usual properties of eigenprojections. It must be noted that $\dot{q}$ (or its incremental counterpart $\Delta q=\Delta t \dot{q}$ ) is a signed scalar factor to the $q_{j}$. In other words, this decomposition can be seen as a reparameterisation of $\mathbf{M}$ in a more convenient way, since it will be seen in the next section that it can be used "as is" in the plastic potential $\varphi^{p}$ and the dissipation pseudo-potential $\psi$.

Eq. (10) has a special meaning: it defines a flow rule for $\dot{\mathbf{F}}^{p}$ and establishes a constraint between $\mathbf{F}^{p}$ and $q$ through the flow directions $q_{i} \mathbf{M}_{i}$. Due to this constraint, $\mathbf{F}^{p}$ becomes a internal variable dependent of the (independent) internal variables $\left\{q, q_{i}, \mathbf{M}_{i}\right\}$. It is worth noting that the flow direction is now described by $\mathbf{M}_{i}$ and $q_{i}$ quantities.

\subsection{Incremental potential and updates}

Consider the decoupled form of the free energy in volumetric and isochoric contributions

$W(\mathscr{E})=U(J)+\varphi^{e}\left(\widehat{\mathbf{C}}^{e}\right)+\varphi^{p}\left(\mathbf{F}^{p}, \mathbf{Q}\right)$

and consider a time discretization with generic time intervals $\left[t_{n}, t_{n+1}\right]$. At the end of a time increment the free energy writes

$W\left(\mathscr{E}_{n+1}\right)=U\left(J_{n+1}\right)+\varphi^{e}\left(\widehat{\mathbf{C}}_{n+1}^{e}\right)+\varphi^{p}\left(\mathbf{F}_{n+1}^{p}, \mathbf{Q}_{n+1}\right)$.

The first term takes into account pure elastic volumetric deformations depending on $J(\mathbf{F})$. A classical (but not unique) expression is

$U(J)=\frac{K}{2}[\ln J]^{2}$.

The elastic potential $\varphi^{e}$ is assumed to be an isotropic function of the elastic deformation depending on its eigenvalues:

$\varphi^{e}\left(\widehat{\mathbf{C}}^{e}\right)=\varphi^{e}\left(c_{1}^{e}, c_{2}^{e}, c_{3}^{e}\right)$

This function is also described in terms of the natural strain $\epsilon^{e}=\frac{1}{2} \ln \widehat{\mathbf{C}}^{e}$ which has the same eigenprojections of $\widehat{\mathbf{C}}^{e}$ and eigenvalues given by $\epsilon_{i}^{e}=\frac{1}{2} \ln c_{i}^{e}$ :

$\varphi^{e}\left(\boldsymbol{\epsilon}^{e}\right)=\varphi^{e}\left(\epsilon_{1}^{e}, \epsilon_{2}^{e}, \epsilon_{3}^{e}\right)$.

Due to Eqs. (8) and (9), the value of $\widehat{\mathbf{C}}_{n+1}^{e}$ depends on $\mathbf{F}_{n+1}^{p}$ at the end of the time increment. Since at the beginning of the interval $\mathbf{F}_{n}^{p}$ is assumed to be known, the incremental update $\mathbf{F}_{n+1}^{p}$ is calculated from the exponential mapping [17]

$\mathbf{F}_{n+1}^{p} \mathbf{F}_{n}^{p-1}=\Delta \mathbf{F}^{p}=\exp \left[\Delta t \mathbf{D}^{p}\right] \Rightarrow \mathbf{F}_{n+1}^{p}=\exp \left[\Delta t \mathbf{D}^{p}\right] \mathbf{F}_{n}^{p}$

Using decomposition (14), $\mathbf{F}_{n+1}^{p}$ can be written in term of the internal variables $\Delta q, q_{i}, \mathbf{M}_{i}$ :

$\mathbf{F}_{n+1}^{p}=\exp \left[\Delta q \sum_{i=1}^{3} q_{i} \mathbf{M}_{i}\right] \mathbf{F}_{n}^{p}$.

The plastic potential $\varphi^{p}$ accounts for hardening phenomena. Just for simplicity, we consider an isotropic hardening and this potential depends solely on the internal variable $q$ :

$\varphi^{p}=\varphi^{p}(q)$,

$q(t)=\int_{0}^{t} \dot{q} \mathrm{~d} t \quad q_{n+1}=q_{n}+\Delta t \dot{q}=q_{n}+\Delta q$.

The dissipative potential is defined to be dependent on the temporal derivative of $q$ and its definition provides an exact penalization for negative values of $\dot{q}$

$\psi\left(\mathbf{D}^{p}\right)=\psi\left(\frac{\Delta q}{\Delta t}\right)=\psi(\dot{q})= \begin{cases}\bar{\psi}(\dot{q}) & \text { if } \dot{q} \geqslant 0, \\ +\infty & \text { if } \dot{q}<0,\end{cases}$

where $\bar{\psi}(\dot{q})$ may take different expressions for positive values of $\dot{q}$.
Due to these choices, the generic set of state (internal and external) variables $\left\{\mathbf{F}, \mathbf{F}^{p}, \mathbf{Q}\right\}$ within the time interval is reduced to the set $\left\{\mathbf{F}, \Delta q, q_{i}, \mathbf{M}_{i}\right\}$. The incremental potential (5) is thus re-written as

$$
\begin{aligned}
& \Psi\left(\mathbf{F}_{n+1} ; \mathscr{E}_{n}\right)= \Psi\left(\mathbf{C}_{n+1} ; \mathscr{E}_{n}\right)=\Delta U\left(J_{n+1}\right) \\
&+\min _{\Delta q, \mathbf{M}_{i}, q_{i}}\left\{\Delta \varphi^{e}\left(\widehat{\mathbf{C}}_{n+1}^{e}\right)+\Delta \varphi^{p}\left(q_{n+1}\right)+\Delta t \bar{\psi}\left(\frac{\Delta q}{\Delta t}\right)\right\}, \\
& \Delta \varphi^{e}\left(\widehat{\mathbf{C}}_{n+1}^{e}\right)=\varphi^{e}\left(\widehat{\mathbf{C}}_{n+1}^{e}\right)-\varphi^{e}\left(\widehat{\mathbf{C}}_{n}^{e}\right), \\
& \Delta \varphi^{p}\left(q_{n+1}\right)=\varphi^{p}\left(q_{n+1}\right)-\varphi^{p}\left(q_{n}\right) \\
& \Delta U\left(J_{n+1}\right)=U\left(J_{n+1}\right)-U\left(J_{n}\right),
\end{aligned}
$$

where the minimization operation is constrained by the conditions

$q_{i} \in K_{Q}=\left\{p_{i} \in \mathbb{R}: \sum_{i=1}^{3} p_{i}=0 ; \sum_{i=1}^{3} p_{i}^{2}=3 / 2\right\}$,

$\mathbf{M}_{i} \in K_{M}=\left\{\mathbf{N}_{i} \in \operatorname{Sym}: \mathbf{N}_{i} \cdot \mathbf{N}_{i}=1, \quad \mathbf{N}_{i} \cdot \mathbf{N}_{j}=0, \quad i \neq j\right\}$,

$\Delta q \geqslant 0$

First order necessary optimality conditions of the minimization problem (25) take into account the derivatives of potential $\Psi$ as well as the derivatives of constraints (29)-(31). The minimization along the eigenprojections $\mathbf{M}_{i}$ can be performed analytically. To this aim, a relevant relation between elastic and plastic deformations is shown:

$$
\begin{aligned}
& \widehat{\mathbf{F}}_{n+1}^{e}=\widehat{\mathbf{F}}_{n+1} \widehat{\mathbf{F}}_{n+1}^{p-1}=\widehat{\mathbf{F}}^{p r}\left(\exp \left[\Delta t \mathbf{D}^{p}\right]\right)^{-1}, \quad \widehat{\mathbf{F}}^{p r}=\widehat{\mathbf{F}}_{n+1} \mathbf{F}_{n}^{p-1}, \\
& \widehat{\mathbf{C}}_{n+1}^{e}=\widehat{\mathbf{F}}_{n+1}^{e T} \widehat{\mathbf{F}}_{n+1}^{e}=\widehat{\mathbf{C}}^{p r}\left(\exp \left[\Delta t \mathbf{D}^{p}\right]\right)^{-2}, \quad \widehat{\mathbf{C}}^{p r}=\mathbf{F}_{n}^{p-T} \widehat{\mathbf{C}}_{n+1} \mathbf{F}_{n}^{p-1}, \\
& \boldsymbol{\epsilon}_{n+1}^{e}=\frac{1}{2} \ln \widehat{\mathbf{C}}_{n+1}^{e}=\boldsymbol{\epsilon}^{p r}-\Delta t \mathbf{D}^{p}, \quad \boldsymbol{\epsilon}^{p r}=\frac{1}{2} \ln \widehat{\mathbf{C}}^{p r},
\end{aligned}
$$

where the superscript " $p r$ " is the usual notation for a predictor quantity, relating values known at time $t_{n+1}$ (i.e., $\widehat{\mathbf{F}}_{n+1}, \widehat{\mathbf{C}}_{n+1}$ ) with values known at time $t_{n}$ (i.e., $\mathbf{F}_{n}^{p}$ ). Eq. (33) is only valid if co-linearity between $\widehat{\mathbf{C}}^{p r}$ and $\mathbf{D}^{p}$ is assumed in order to allow permutation between both tensors. Using this ansatz, it is shown in the Appendix that the minimization with respect to $\mathbf{M}_{i}$ is achieved when the tensors $\widehat{\mathbf{C}}_{n+1}^{e}$, $\widehat{\mathbf{C}}^{p r}$ and $\mathbf{D}^{p}$ share the same eigenprojections: $\mathbf{E}_{i}^{e}=\mathbf{E}_{i}^{p r}=\mathbf{M}_{i}$. This means collinearity between $\widehat{\mathbf{C}}^{p r}$ and $\mathbf{D}^{p}$ which corroborates the permutability assumption made in (33).

On the other hand, the necessary optimality conditions for $q$ and $\Delta q$ take the form

$r_{i}=-\frac{\partial \Delta \varphi^{e}}{\partial \epsilon_{i}^{e}} \Delta q+\lambda+2 \beta q_{i}=0, \quad i=1,2,3$,

$r_{4}=-\sum_{i=1}^{3} \frac{\partial \Delta \varphi^{e}}{\partial \epsilon_{i}^{e}} q_{i}+\frac{\partial \Delta \varphi^{p}}{\partial \Delta q}+\frac{\partial \psi}{\partial \dot{q}} \geqslant 0$

$r_{5}=\sum_{i=1}^{3} q_{i}=0$,

$r_{6}=\sum_{i=1}^{3} q_{i}^{2}=3 / 2$

$\Delta q \geqslant 0$,

$r_{4} \Delta q=0$.

Once the minimization is performed, the derivative of $\Psi$ with respect to $\widehat{\mathbf{C}}_{n+1}$ and $J_{n+1}$ should be calculated in order to obtain the Piola-Kirchhoff stress tensor. Due to the separation of potential $\Psi$ in isochoric and volumic contributions, the stress tensor $\mathbf{P}$ is rewritten as

$$
\begin{aligned}
\mathbf{P}_{n+1} & =2 \mathbf{F}_{n+1} \frac{\partial \Psi\left(\mathbf{C}_{n+1} ; \mathscr{E}_{n}\right)}{\partial \mathbf{C}_{n+1}} \\
& =\mathbf{F}_{n+1}\left[J_{n+1}^{-2 / 3} \operatorname{DEV}\left(2 \frac{\partial \varphi^{e}}{\partial \widehat{\mathbf{C}}_{n+1}}\right)+\frac{\partial U}{\partial J_{n+1}} J_{n+1} \mathbf{C}_{n+1}^{-1}\right],
\end{aligned}
$$


where (see Appendix for operational details):

$\left.\frac{\partial \varphi^{e}}{\partial \widehat{\mathbf{C}}_{n+1}}=\left(\sum_{i=1}^{3} \frac{\partial \varphi^{e}}{\partial c_{i}^{p r}} \frac{\partial c_{i}^{p r}}{\partial \widehat{\mathbf{C}}^{p r}}\right) \frac{\partial \widehat{\mathbf{C}}^{p r}}{\partial \widehat{\mathbf{C}}_{n+1}}=\mathbf{F}_{n}^{p-1} \sum_{i=1}^{3} \frac{\partial \varphi^{e}}{\partial \epsilon_{i}^{e}} \frac{1}{2 c_{i}^{p r}} \mathbf{E}_{i}^{p r}\right) \mathbf{F}_{n}^{p-\mathrm{T}}$.

\subsection{Material tangents}

An important aspect from the numerical implementation point of view is the determination of the tangent matrix, consistent with the constitutive incremental update algorithm. The contribution to the tangent matrix from geometric terms is common to any hyperelastic model. Thus, we focus here on the expression of the second derivative of the present incremental material update. We will use here the notation $\frac{\mathrm{d}(\cdot)}{\mathrm{d}_{n+1}}$ as the total derivative of the argument with respect $\widehat{\mathbf{C}}_{n+1}$. We define thus the tensor $\mathscr{C}$ :

$\mathscr{C}=\frac{\mathrm{d}}{\mathrm{d} \widehat{\mathbf{C}}_{n+1}}\left(\frac{\mathrm{d} \Psi}{\mathrm{d} \widehat{\mathbf{C}}_{n+1}}\right)=\frac{\mathrm{d}}{\mathrm{d} \widehat{\mathbf{C}}_{n+1}}\left(\frac{\partial \varphi^{e}}{\partial \widehat{\mathbf{C}}_{n+1}}\right)$.

Considering $\widehat{C}^{p r}=\left(\widehat{\mathbf{F}}^{p}\right)_{n}^{-\mathrm{T}} \widehat{\mathbf{C}}_{n+1}\left(\widehat{\mathbf{F}}^{p}\right)_{n}^{-1}$, calling $\mathbf{f}^{p n}=\left(\widehat{\mathbf{F}}^{p}\right)_{n}^{-1}$ and dropping index $n+1$, we have: (see Appendix):

$\mathscr{C}_{i j k l}=\sum_{m, t, p, q=1}^{3} \mathbf{f}_{i m}^{p n} \mathbf{f}_{j t}^{p n} \frac{\mathrm{d}}{\mathrm{d} \widehat{\mathbf{C}}_{p q}^{p r}}\left(\frac{\partial \varphi^{e}}{\partial \widehat{\mathbf{C}}_{m t}^{p r}}\right) \mathbf{f}_{k p}^{p n} \mathbf{f}_{l q}^{p n}=\mathscr{C}_{k l i j}^{\varphi^{e}}=\mathscr{C}_{j i k l}^{\varphi^{e}}$.

The critical point is the obtention of the derivatives of $\varphi^{e}$ with respect to $\widehat{\mathbf{C}}^{p r}=c_{i}^{p r} \mathbf{E}_{i}^{p r}$. In spectral coordinates this requires the computation of the following functions (see Appendix for details)

$y_{i}=\frac{\partial \varphi^{e}}{\partial c_{i}^{p r}}=\frac{\partial \varphi^{e}}{\partial \epsilon_{i}^{e}} \frac{1}{2 c_{i}^{p r}}$,

$y_{i, j}=\frac{\mathrm{d}}{\mathrm{d} c_{j}^{p r}}\left(\frac{\partial \varphi^{e}}{\partial \epsilon_{i}^{e}} \frac{1}{2 c_{i}^{p r}}\right)=\frac{\partial^{2} \varphi^{e}}{\partial \epsilon_{i}^{e} \partial \epsilon_{i}^{e}} \frac{\mathrm{d} \epsilon_{i}^{e}}{\mathrm{~d} \epsilon_{j}^{p r}} \frac{1}{4 c_{i}^{p r} c_{j}^{p r}}-\frac{\partial \varphi^{e}}{\partial \epsilon_{i}^{e}} \frac{\delta_{i j}}{2\left(c_{i}^{p r}\right)^{2}}$.

The terms $\frac{\partial \varphi^{e}}{\partial \epsilon_{k}^{e}}$ and $\frac{\partial^{2} \varphi^{e}}{\partial \epsilon_{k}^{\partial} \epsilon_{l}^{e}}$ are straightforward. On other hand, the relation $\epsilon_{k}^{e}\left(\epsilon_{1}^{p r}, \epsilon_{2}^{p r}, \epsilon_{3}^{p r}\right)$ is defined by the derivation of the nonlinear system ((35) and (38), see Appendix) which provides the terms $\frac{\mathrm{d} \epsilon_{i}^{e}}{\mathrm{~d} \epsilon_{j}}$.

\section{Minimization strategy}

The first information to be obtained from the minimization operation is the characterization of the plastic or elastic nature of the incremental step. The proposed decomposition of the plastic stretching $\mathbf{D}^{p}$ allows for a clear visualization of this issue. Indeed, while Eq. (35) provide the variations of the potential function with respect to the eigenvalues $q_{i}$ along a circle of radius $\sqrt{3 / 2}$ (condition (38)) on the deviatoric plane (condition (37)), Eq. (36) computes the derivative of this potential due to the plastic increment $\Delta q$. A plastic increment occur when the minimum is achieved for a $\Delta q>0$. Conversely if the minimizer is such that $\Delta q=0$ then, the incremental step is elastic. A null minimizer $\Delta q$ is obtained if the derivative. $\left.r_{4}\right|_{\Delta q=0} \geqslant 0$ for all possible values of $q_{i}$. In other words, an elastic step occur if the minimum value of $r_{4}$ at $\Delta q=0$ is non-negative. Mathematically, it is necessary to solve the problem

$$
\begin{aligned}
& \left.\min _{q_{i}}-\sum_{j=1}^{3} \frac{\partial \Delta \varphi^{e}}{\partial \epsilon_{i}^{e}} q_{i}+\frac{\partial \Delta \varphi^{p}}{\partial \Delta q}+\frac{\partial \psi}{\partial \dot{q}}\right), \\
& q_{i} \in K_{Q}=\left\{p_{i} \in \mathbb{R}: \sum_{i=1}^{3} p_{i}=0 ; \sum_{i=1}^{3} p_{i}^{2}=3 / 2\right\} .
\end{aligned}
$$

Using a Lagrangian function

$$
\begin{aligned}
\widetilde{\mathscr{L}}\left(\Delta q, q_{i}\right)= & \left.-\sum_{i=1}^{3} \frac{\partial \Delta \varphi^{e}}{\partial \epsilon_{i}^{e}} q_{i}+\frac{\partial \Delta \varphi^{p}}{\partial \Delta q}+\frac{\partial \psi}{\partial \dot{q}}\right)+\tilde{\lambda}\left(\sum_{i=1}^{3} q_{i}\right) \\
& \left.+\tilde{\beta} \sum_{i=1}^{3} q_{i}^{2}-3 / 2\right),
\end{aligned}
$$

the necessary first order optimality conditions are easily written:

$-\frac{\partial \Delta \varphi^{e}}{\partial \epsilon_{i}^{e}}+\tilde{\lambda}+2 \tilde{\beta} q_{i}=0 \quad(i=1,2,3)$,

$\sum_{i=1}^{3} q_{i}=0$

$\sum_{i=1}^{3} q_{i}^{2}-3 / 2=0$

This problem is easily solved analytically. Summing up the three equations in (49) and using (50) it comes that $\tilde{\lambda}=1 / 3 \sum_{i=1}^{3} \frac{\partial \Delta \varphi^{e}}{\partial \epsilon_{i}^{e}}$ $=1 / 3 \operatorname{tr}\left(\frac{\partial \Delta \varphi^{e}}{\partial \epsilon^{e}}\right)$. Introducing this expression in (49) and calling $\frac{\partial \Delta \varphi^{e}}{\partial \epsilon_{i}^{e}}=\frac{\partial \Delta \varphi^{e}}{\partial \epsilon_{i}^{e}}-1 / 3 \operatorname{tr}\left(\frac{\partial \Delta \varphi^{e}}{\partial \epsilon^{e}}\right)$ we obtain, after algebraic operations, that

$q_{i}=\sqrt{\frac{3}{2}} \frac{\frac{\widehat{\partial \Delta \varphi^{e}}}{\partial \epsilon_{i}^{e}}}{\left\|\frac{\widehat{\partial \Delta \varphi^{e}}}{\partial \epsilon^{e}}\right\|}, \quad$ where $\left\|\frac{\partial \widehat{\Delta \varphi^{e}}}{\partial \epsilon^{e}}\right\|^{2}=\sum_{i=1}^{3}\left(\frac{\partial \widehat{\Delta \varphi^{e}}}{\partial \epsilon_{i}^{e}}\right)^{2}$.

Substituting this value on Eq. (36) we arrive to the expected condition for a plastic step: the minimum value of the derivative of $\Psi$ with respect to $\Delta q$ evaluated at $\Delta q=0$ must be negative, i.e.

$-\sigma_{M}+\left.\frac{\partial \Delta \varphi^{p}}{\partial \Delta q}\right|_{\Delta q=0}+\left.\frac{\partial \psi}{\partial \dot{q}}\right|_{\Delta q=0}<0$.

where the following definition for the equivalent stress $\sigma_{M}$ was used:

$\sigma_{M}=\sqrt{\frac{3}{2}\left\|\frac{\partial \widehat{\Delta \varphi^{e}}}{\partial \epsilon^{e}}\right\|}$

It is important to note that if criterion (53) qualifies the minimizing step to be "plastic" $(\Delta q>0)$ then conditions (39) and (40) may be dropped and the minimization is to be carried out just by Eqs. (35)-(38). Moreover, further simplifications may be used; since $\Delta q>0$, a new set of variables $\left\{\tilde{q}_{1}, \tilde{q}_{2}, \tilde{q}_{3}\right\}$ may be defined using the expression $\tilde{q}_{i}=\Delta q q_{i}$. This eliminates $\Delta q$ as a independent variable, and also the constraint on the size of the $q_{i}$ in (29). Details on this technical simplification will be shown in further article.

It is also interesting to note that the equivalent stress $\sigma_{M}$, as defined in (54), also corresponds to a classical equivalent stress computed from Mandel stress tensor:

$\sigma_{M}=\sqrt{\frac{3}{2} \operatorname{dev} \mathbf{S} \cdot \operatorname{dev} \mathbf{S}}$,

where

$\mathbf{S}=\mathbf{F}^{e \mathrm{~T}} \mathbf{P F}^{p \mathrm{~T}}$.

The Mandel stress tensor is defined in the intermediate configuration, and is symmetric in the case of isotropic elasticity. The above derivation thus shows that the above minimization strategy amounts to computing an elastic predictor Mandel stress, evaluate the classical von Mises criterion in terms of this stress tensor, and, if the criterion is verified, apply a plastic corrector step. This process parallels the classical predictor-corrector scheme used in small strains. In general, it may not correspond to a radial return, though, since the predictor $q_{i}$ may be different from the overall optimal ones.

\section{Particular viscoplastic models}

In this section, some expressions for the potentials are specified in order to obtain two particular behaviors: Hencky-based and Ogden-based models. 
Table 1

Material parameters for cyclic traction and shear tests

\begin{tabular}{llll}
\hline Potential & Ogden & & Hencky \\
\hline$\mu_{i}$ & 0.7 & -0.7 & $\mu=20$ \\
$\alpha_{i}$ & 5 & -5 & \\
\hline
\end{tabular}

As already said, the dissipation pseudo-potential $\psi$ defined by (24) plays the important role of enforcing the positiveness of $\dot{q}$. In addition, the function $\bar{\psi}(\dot{q})$ defines the rate dependence or independence of the plastic deformation. In the case of rate-independent plasticity we have

$\bar{\psi}(\dot{q})=Y_{0} \dot{q}$

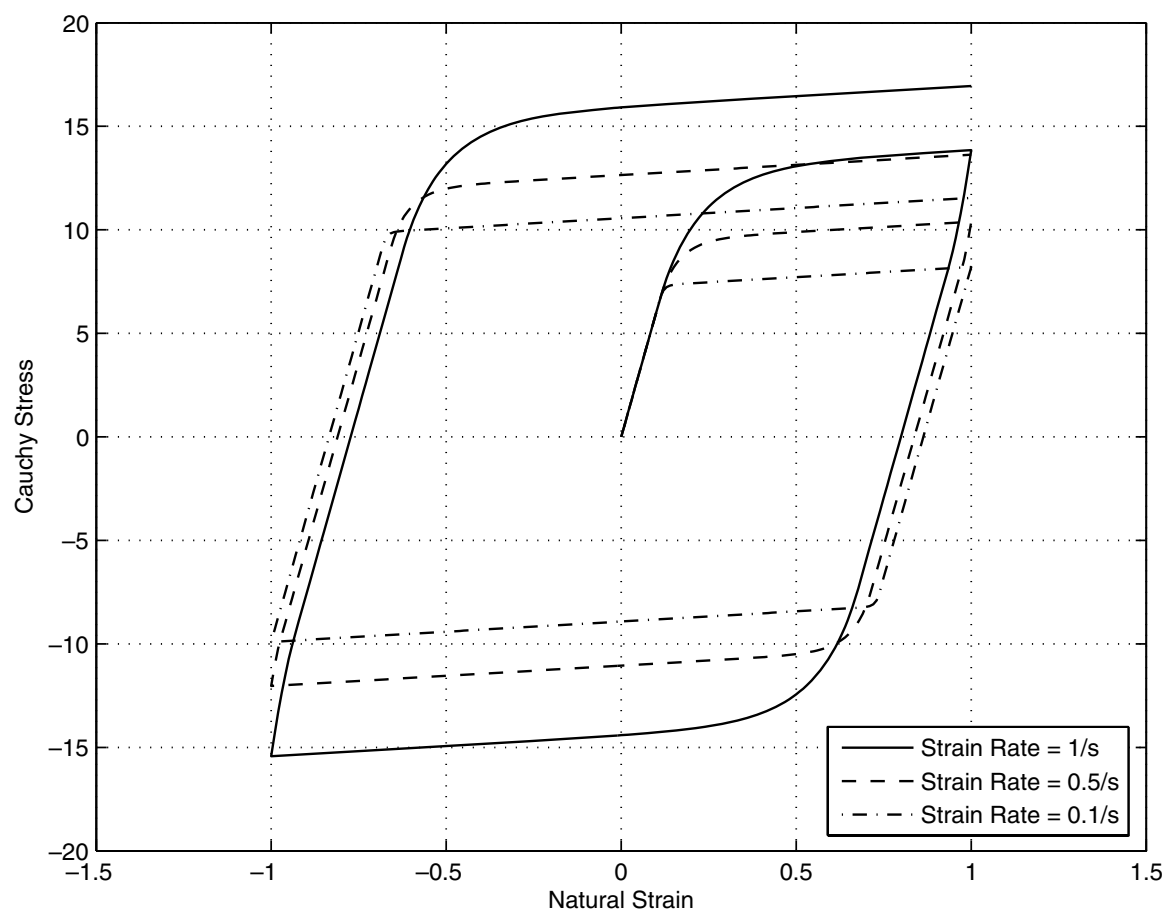

Fig. 1. Traction test in Hencky model. Axial stress $\sigma_{x}$.

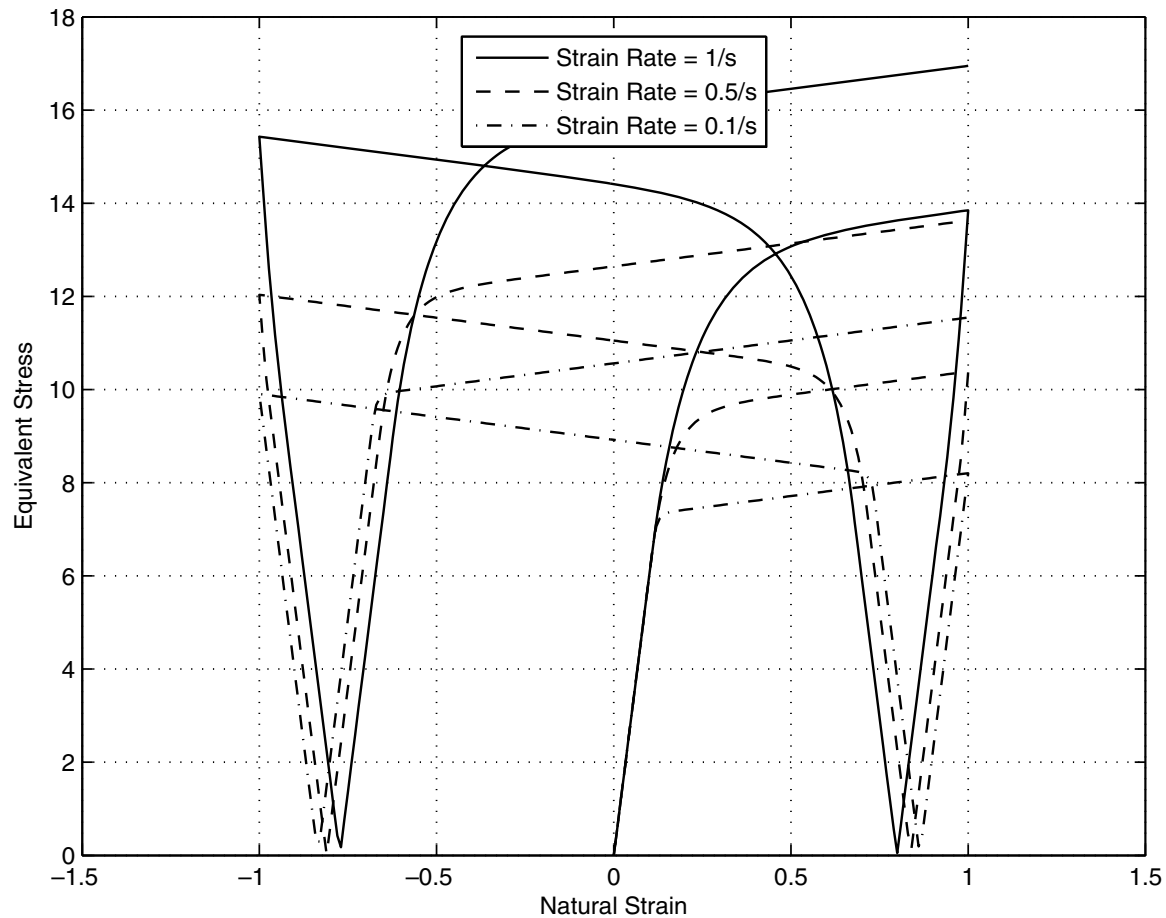

Fig. 2. Traction test in Hencky model. Equivalent stress $\sigma_{M}$. 


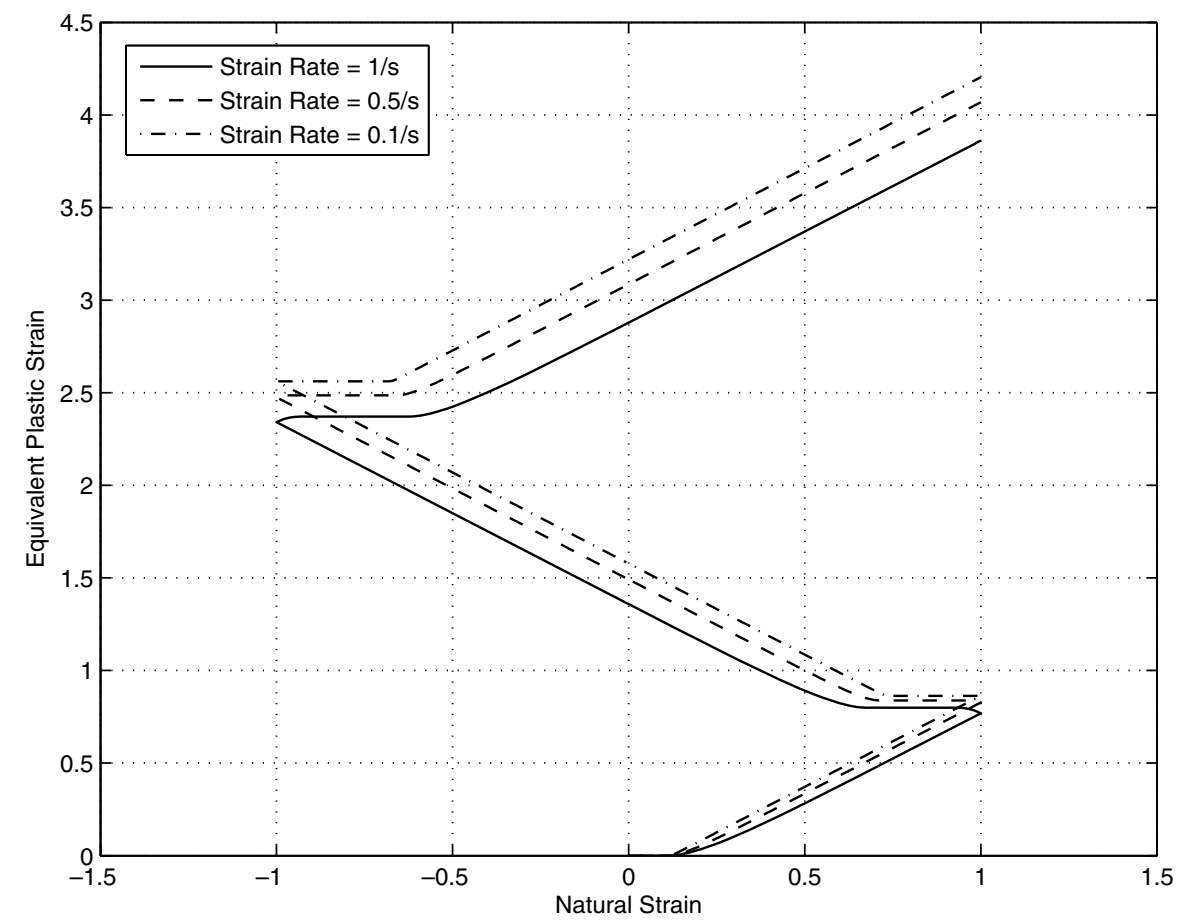

Fig. 3. Traction test in Hencky model. Equivalent plastic strain $q$.

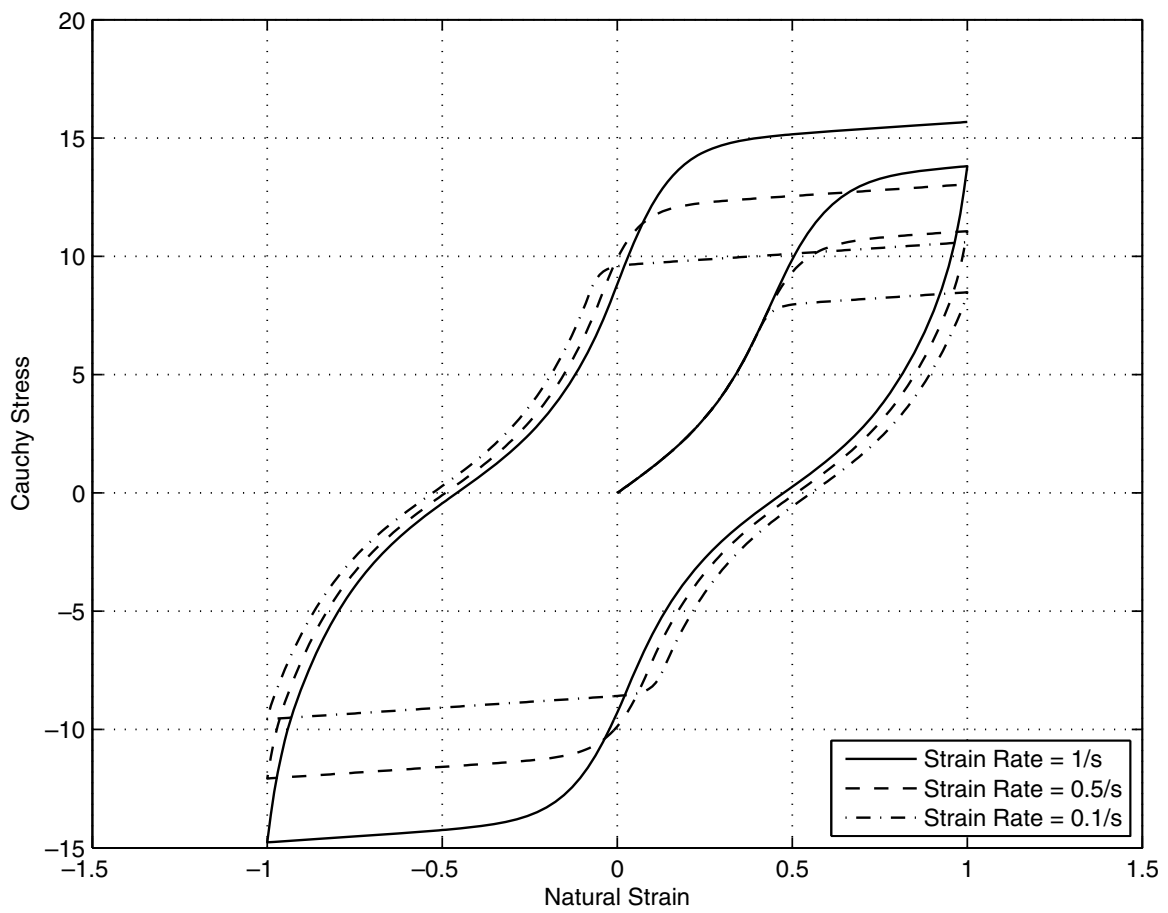

Fig. 4. Traction test in Ogden model. Axial stress $\sigma_{x}$.

or, in a Perzyna rate-dependent law,

$\bar{\psi}(\dot{q})=\frac{m Y_{0} \dot{q}_{0}}{m+1}\left(\frac{\dot{q}}{\dot{q}_{0}}\right)^{\frac{m+1}{m}}$.

The (isotropic) hardening rule is provided by $\varphi^{p}$. A quite generic expression for this potential may be given by
$\varphi^{p}(q)=\Sigma_{0} q+\frac{1}{2} H q^{2}+\mu^{p}\left(q+\frac{1}{\alpha^{p}} \exp \left(-\alpha^{p} q\right)\right)+\sum_{j=1}^{N} \frac{\mu_{j}^{p}}{\alpha_{j}^{p}+1}(q)^{\alpha_{j}^{p}+1}$,

where $\Sigma_{0}, H, \mu^{p}, \alpha^{p}, \mu_{j}^{p}$ and $\alpha_{j}^{p}$, are material parameters. Linear hardening is given by the first two terms, depending on $\Sigma_{0}$ and $H$. The 


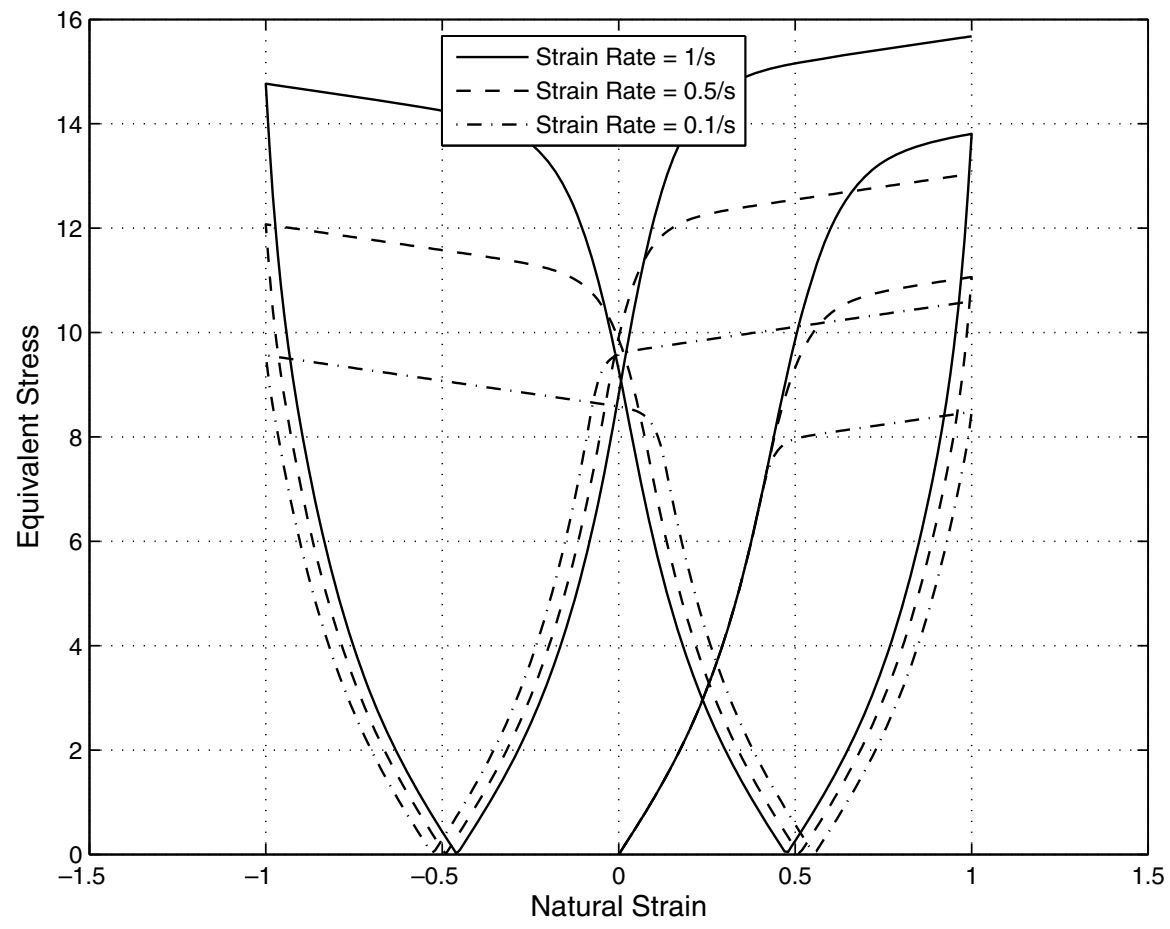

Fig. 5. Traction test in Ogden model. Equivalent stress $\sigma_{M}$.

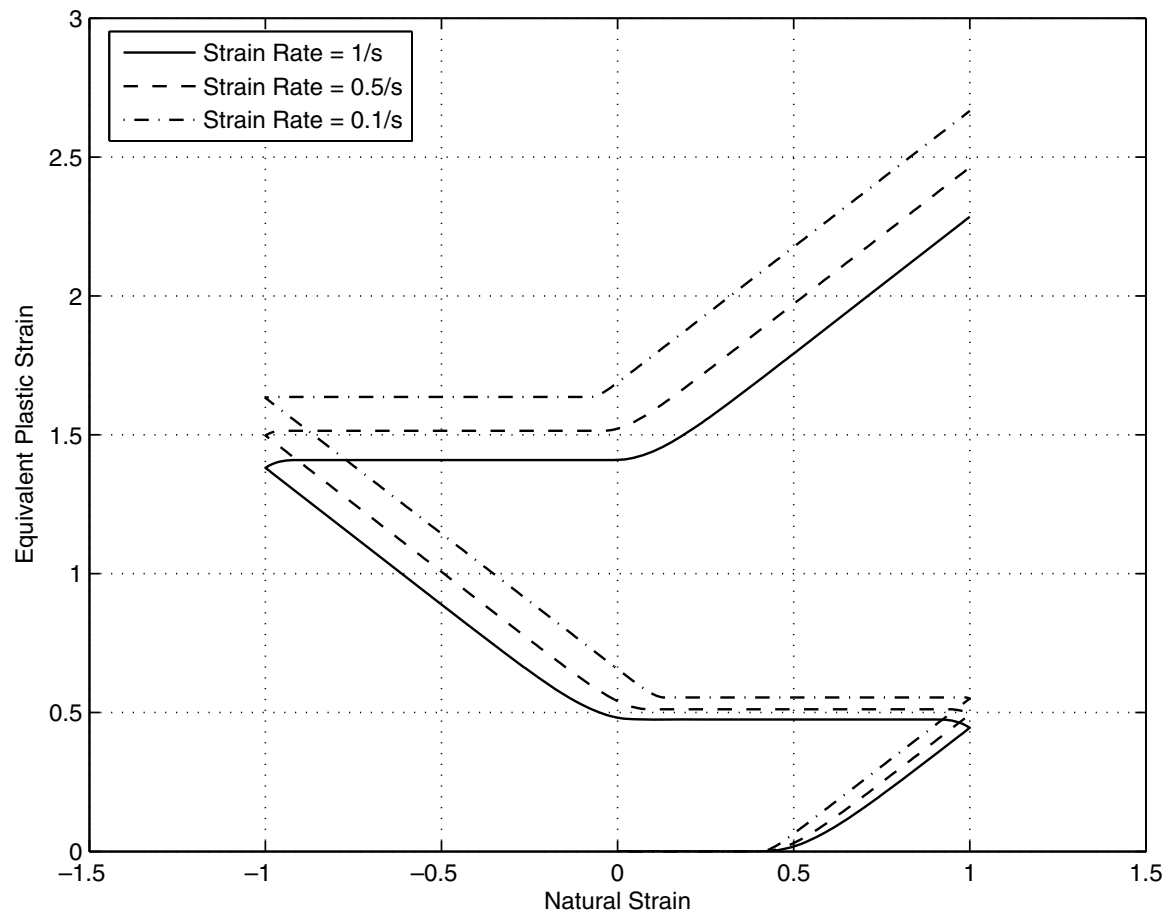

Fig. 6. Traction test in Ogden model. Equivalent plastic strain $q$.

third term provides a saturation behavior, controlled by $\alpha^{p}$ and $\mu^{p}$. Finally, the fourth term is an extension of the Ramber-Osgood law, which considers just a single term within the sum, i.e., $N=1$, and parameters $\alpha_{1}^{p}$ and $\mu_{1}^{p}$.

Note that hardening can also be introduced in the dissipation pseudo-potential, e.g. through a dependence $Y_{0}(q)$ in (57). But since the partition of hardening between dissipation pseudo-potential and plastic free energy only becomes relevant in a ther- mo-mechanical setting, we will limit hardening terms to the latter.

\subsection{Hencky-based model}

In this section we analyze the case when the elastic potential is based on a quadratic form of the logarithmic strain tensor (Hencky-type potentials $[18,16])$ : 
$\varphi^{e}=\mu^{e} \sum_{i=1}^{3}\left(\epsilon_{i}^{e}\right)^{2}$.

For simplicity reasons, we assume that $\bar{\psi}$ and $\varphi^{p}$ take the forms (57) and the first two terms of (59) respectively (rate-independent linear isotropic hardening). Thus,

$$
\begin{aligned}
& \frac{\partial \varphi^{e}}{\partial \epsilon_{i}^{e}}=2 \mu^{e} \epsilon_{i}^{e}=2 \mu^{e}\left(\epsilon_{i}^{p r}-\Delta q q_{i}\right), \\
& \frac{\partial \bar{\psi}}{\partial \dot{q}}=Y_{0}, \quad \frac{\partial \varphi^{p}}{\partial \Delta q}=\Sigma_{0}+H q_{n+1} .
\end{aligned}
$$

It is easily seen that in this case the qualifying conditions (53) reduces to

$-\sigma_{M}+\left(Y_{0}+\Sigma_{0}\right)<0$,

$\sigma_{M}=\sqrt{\frac{3}{2}}\left\|\mathbf{s}^{p r}\right\|, \quad\left\|\mathbf{s}^{p r}\right\|^{2}=\sum_{j=1}^{3}\left(s_{j}^{p r}\right)^{2}, \quad s_{j}^{p r}=2 \mu^{e} \epsilon_{j}^{p r}$,

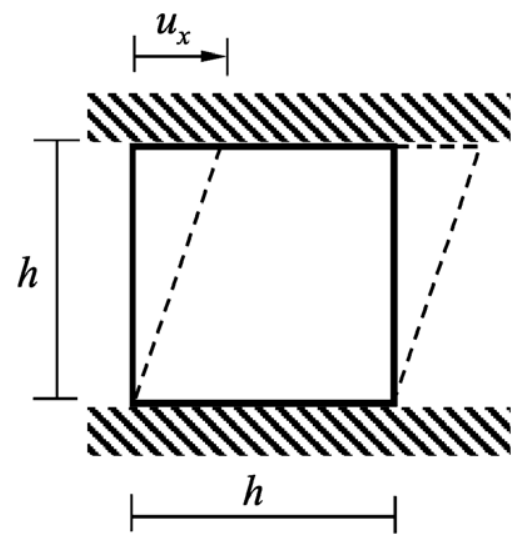

Fig. 7. Shear test. while conditions (35) and (36) take the particular form

$r_{i}=-2 \mu^{e}\left(\epsilon_{i}^{p r}-\Delta q q_{i}\right) \Delta q+\lambda+2 \beta q_{i}=0, \quad i=1,2,3$,

$r_{4}=-\sum_{i=1}^{3} 2 \mu^{e}\left(\epsilon_{i}^{p r}-\Delta q q_{i}\right) q_{i}+\Sigma_{0}+H q_{n+1}+Y_{0}=0$.

After some algebra, it is shown that (see Appendix)

$\Delta q=\frac{\sqrt{\frac{3}{2}}\left\|\mathbf{s}^{p r}\right\|-\left(Y_{0}+\Sigma_{0}+H q_{n}\right)}{3 \mu^{e}+H}$ if $\Delta q \geqslant 0$,

$q_{i}=\frac{3 \mu^{e} \epsilon_{i}^{p r}}{a \Delta q+b}, \quad a=3 \mu^{e}+H, \quad b=\Sigma_{0}+H q_{n}+Y_{0}$,

which is the usual expression for elastoplastic radial return vonMises model. Finally, (65) allow the computation of $\epsilon_{i}^{e}=2 \mu^{e}\left(\epsilon_{i}^{p r}-\Delta q q_{i}\right)$ needed for the elastic potential.

\subsection{Ogden-based model}

In the previous case, the quadratic function of the logarithmic strains is particularly convenient to obtain an explicit expression for the minimizing argument $\Delta q_{j}^{p}$. In spite of this advantage, it is well known that this type of hyperelastic potential do not fit very well other materials like polymers. For that case, a more adequate choice may be the Ogden model $([18,16])$

$\varphi^{e}=\sum_{i=1}^{3} \sum_{p=1}^{N} \frac{\mu_{p}^{e}}{\alpha_{p}}\left(\left[\exp \left(\epsilon_{i}^{e}\right)\right]^{\alpha_{p}}-1\right)$,

$\frac{\partial \varphi^{e}}{\partial \epsilon_{i}^{e}}=\sum_{p=1}^{N} \mu_{p}^{e}\left[\exp \left(\epsilon_{i}\right)\right]^{\alpha_{p}}$.

This model has the property of generalizing others well-known models. For example, Mooney-Rivlin model is achieved taking $N=2, \mu_{1}=2 C_{1}, \mu_{2}=-2 C_{2}$ and $\alpha_{1}=2, \alpha_{2}=-2$.

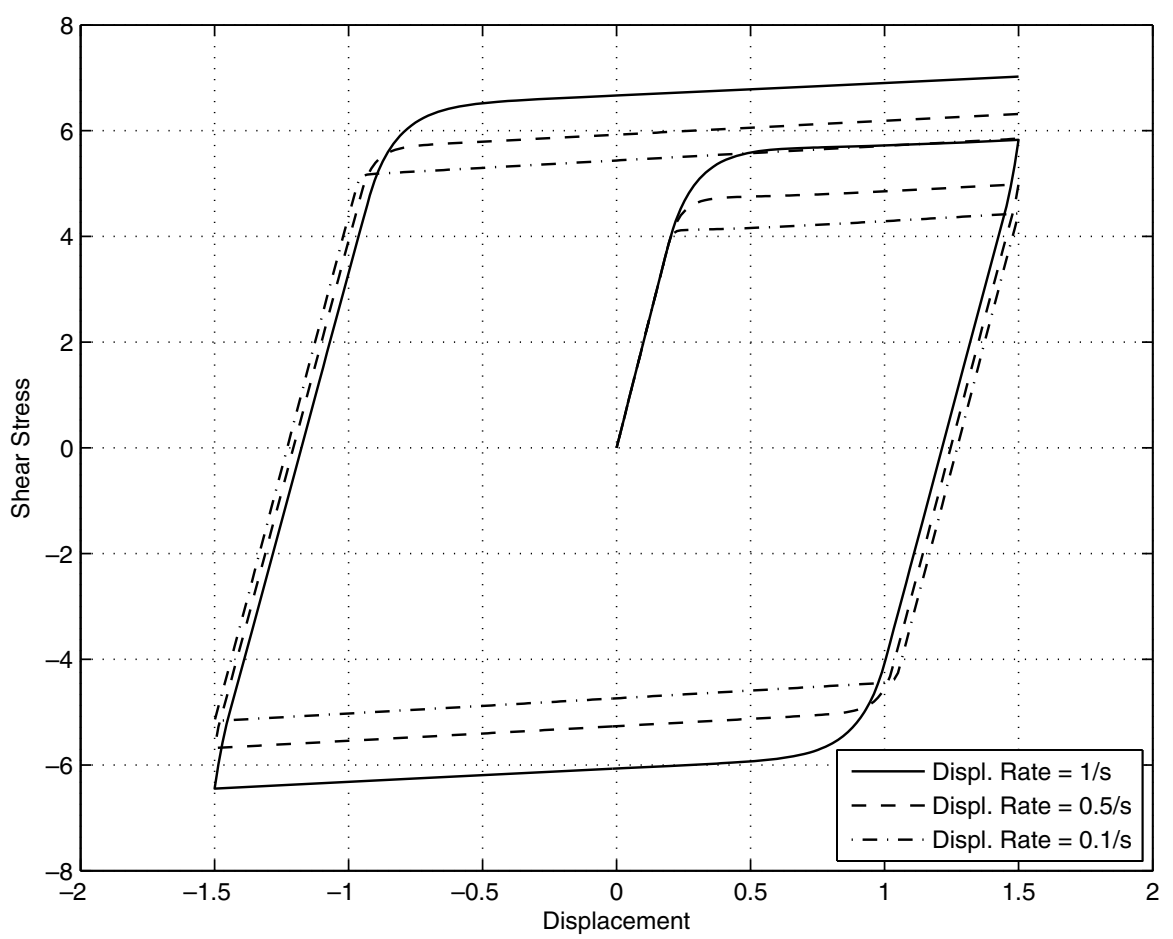

Fig. 8. Shear test in Hencky model. Shear stress $\sigma_{x y}$. 


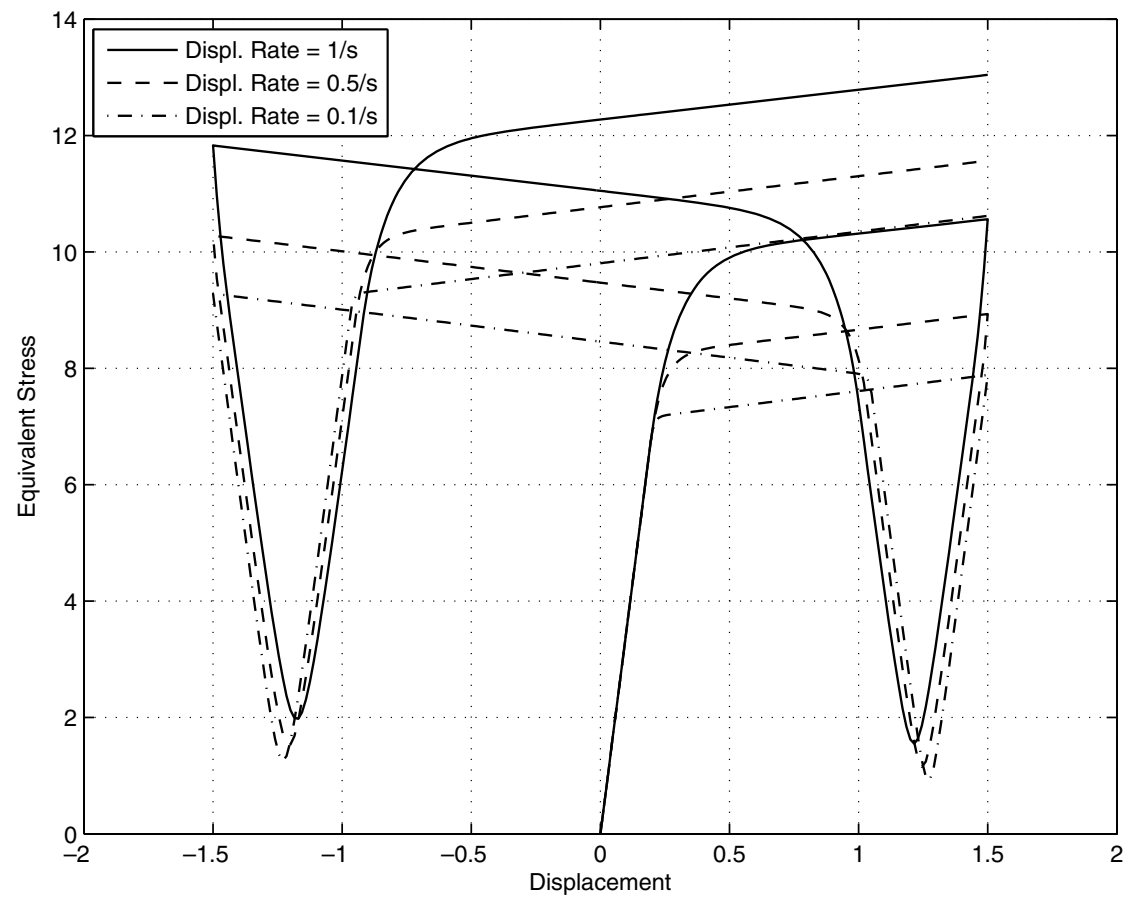

Fig. 9. Shear test in Hencky model. Equivalent stress $\sigma_{M}$.

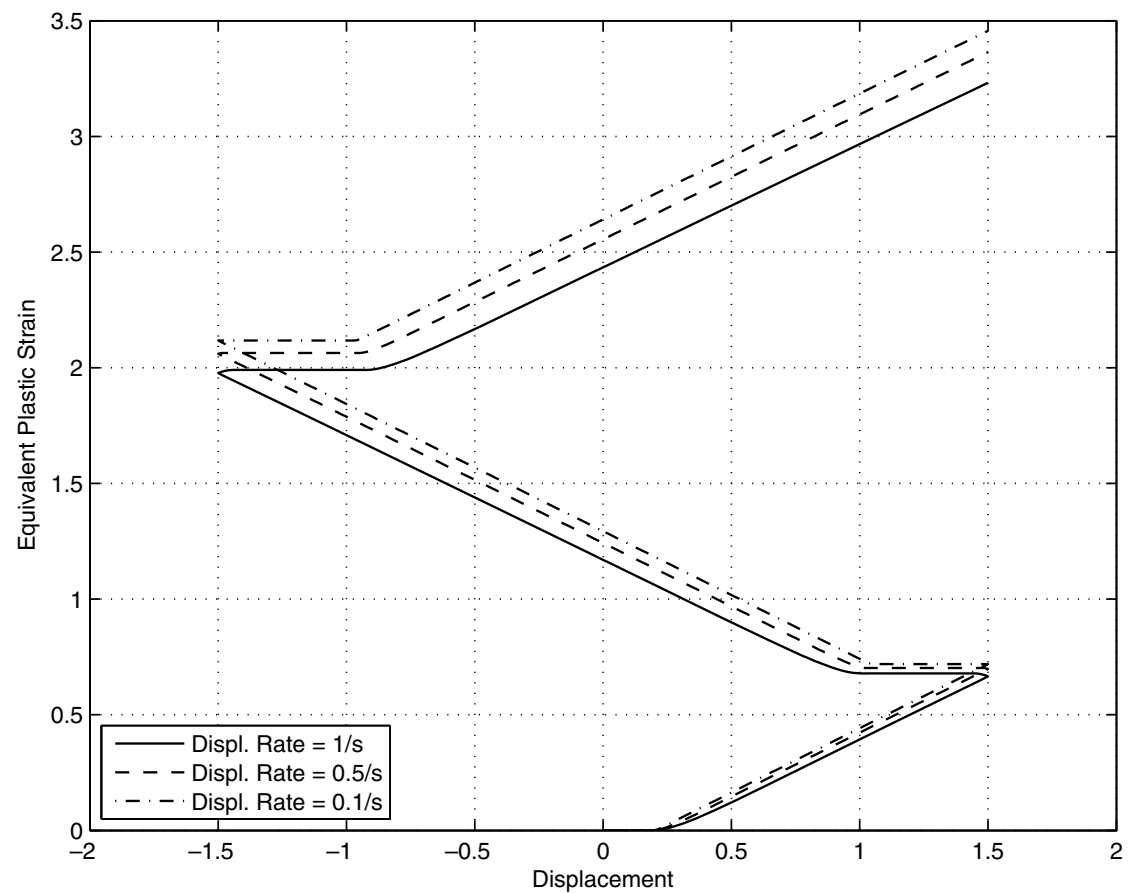

Fig. 10. Shear test in Hencky model. Equivalent plastic strain $q$.

In the present case, conditions (35) and (36) take the expression

$r_{i}=-\sum_{p=1}^{N} \mu_{p}^{e}\left[\exp \left(\epsilon_{i}\right)\right]^{\alpha_{p}} \Delta q+\lambda+2 \beta q_{i}=0, \quad i=1,2,3$,

$r_{4}=-\sum_{p=1}^{N} \mu_{p}^{e}\left[\exp \left(\epsilon_{i}\right)\right]^{\alpha_{p}} q_{i}+\Sigma_{0}+H q_{n+1}+Y_{0}=0$

whose solution provides $\Delta q, q_{i}, \lambda$ and $\beta$.

\section{Numerical examples}

\subsection{Uniaxial traction test}

This simple example illustrates the behavior of this approach for the case of a traction sample submitted to a constant strain rate. Two material models were tested: Hencky and Ogden. Both materials were considered incompressible through a convenient 


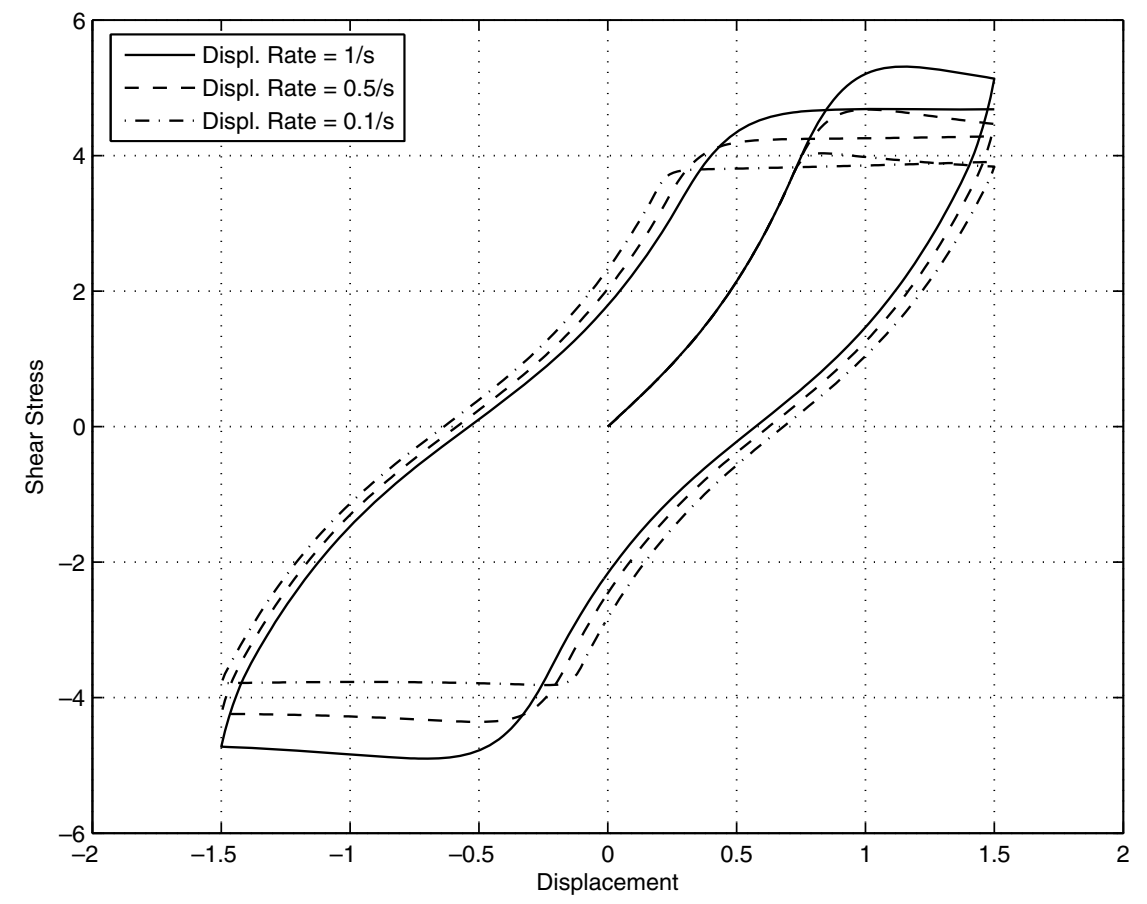

Fig. 11. Shear test in Ogden model. Axial stress $\sigma_{x y}$.

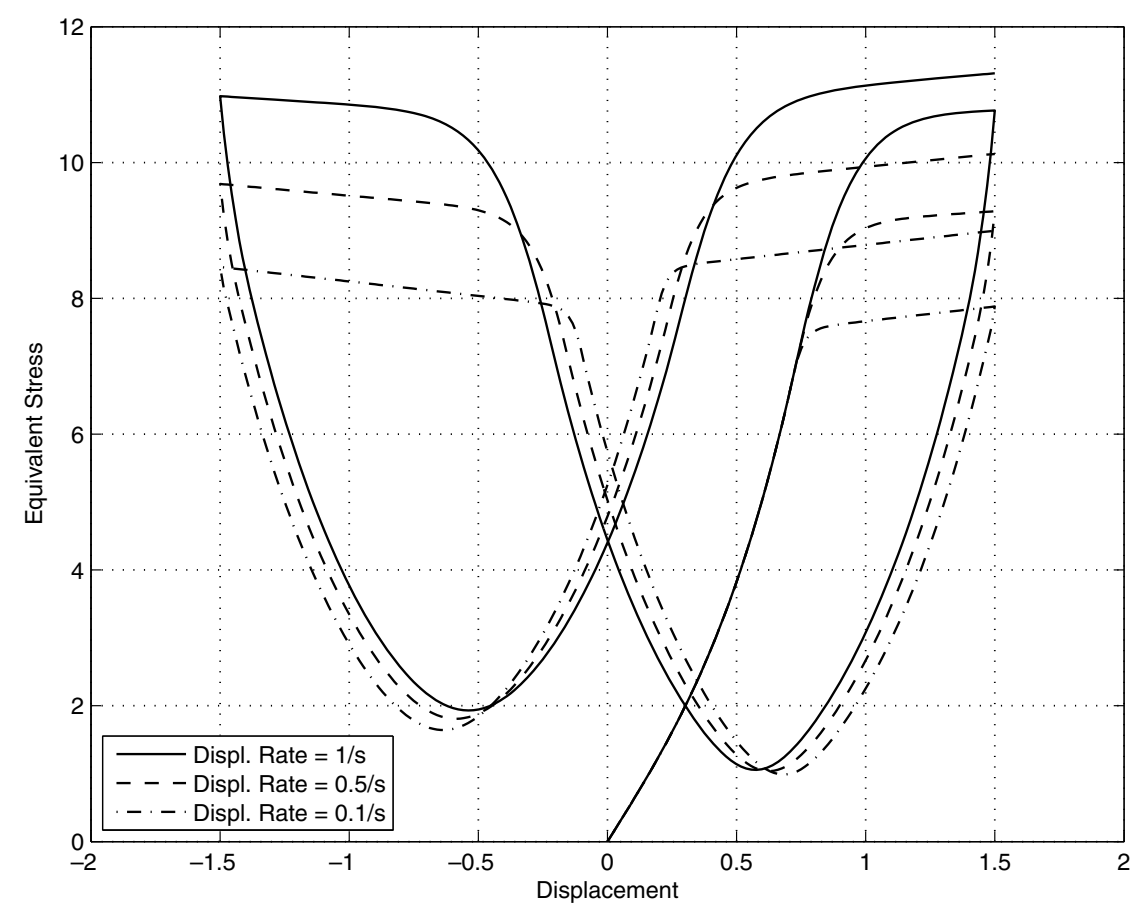

Fig. 12. Shear test in Ogden model. Equivalent stress $\sigma_{M}$.

penalization value of $K$. The Ogden model used $N=2$, i.e., $p=1,2$. The corresponding values are listed in Table $1 .{ }^{4}$ For both cases, potentials (58) and (59) were used with parameters $\Sigma_{0}=7$, $m=1.2, \dot{q}_{0}=1.1, Y_{0}=7, H=1$. It is important to remark that these

\footnotetext{
${ }^{4}$ It is worth to noting that the parameters of 1 do not correspond exactly to the Mooney-Rivlin case, since the exponent $\alpha_{1}$ and $\alpha_{2}$ are different from the number 2 .
}

values are merely illustrative, with no relation to a specific material. Both specimens were elongated and compressed up to $\varepsilon=\ln \lambda=[-1.0,1.0]$ with constant logarithmic strain rates of $1 / s, 0.5 / s$ and $0.1 / s$. Fig. 1 shows the axial Cauchy stress $\sigma_{x}$, while Fig. 2 shows the equivalent stress $\sigma_{M}$ defined in (54) for the Hencky model. Fig. 3 also shows the evolution of the equivalent plastic strain $\Delta q$ for this case. These figures allow to see the expected dependence of the strain rate in the plastic regime as well as the evolution of the 


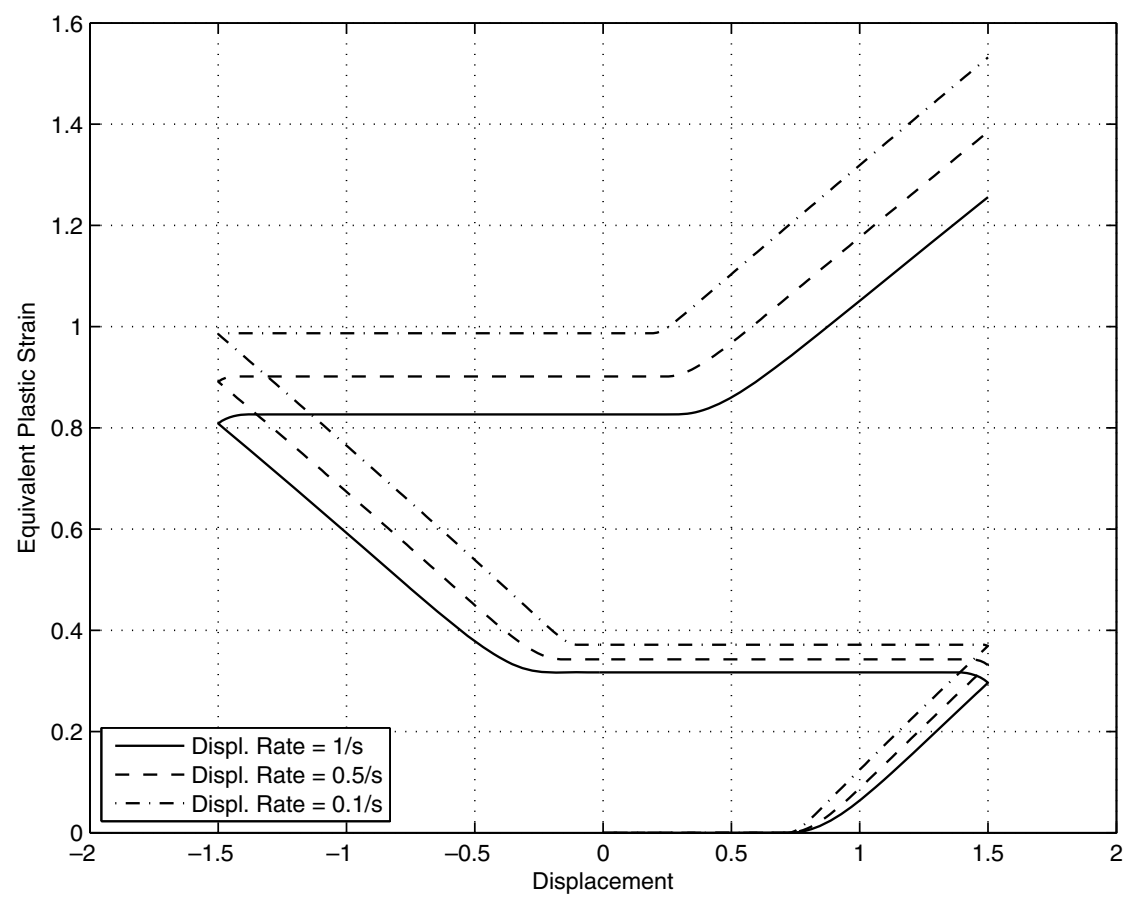

Fig. 13. Shear test in Ogden model. Equivalent plastic strain $q$.

Table 2

Material parameters

\begin{tabular}{lccc}
\hline Potential $\varphi^{e}$ & Ogden & & \\
\hline$\mu_{i}$ & -942.25 & 1404.2 & 352.1 \\
$\alpha_{i}$ & 3.0559 & 1.3328 & 3.8812 \\
Potential $\varphi^{p}$ & Polynomial & Potential $\psi$ & Perzyna \\
\hline$\Sigma_{0}$ & 10 & $Y_{0}$ & 5 \\
$\mu_{1}^{p}$ & 20 & $m$ & 3 \\
$\alpha_{1}^{p}$ & 4.5 & $\dot{q}_{0}$ & 5 \\
\hline
\end{tabular}

yield limit. Figs. 4-6 show analogous results for the Ogden model. It is possible to see a clear difference of behavior in the elastic range.

\subsection{Shear test}

The same materials as in the previous example are submitted to a shear test. The top surface of a cube of unit length (Fig. 7, $h=1$ ) is submitted to a lateral displacement $u_{x}=[-1.5,1.5]$, with constant velocities $\dot{u}_{x}=1 / s, 0.5 / s$, and $0.1 / s$. The evolution of the Cauchy

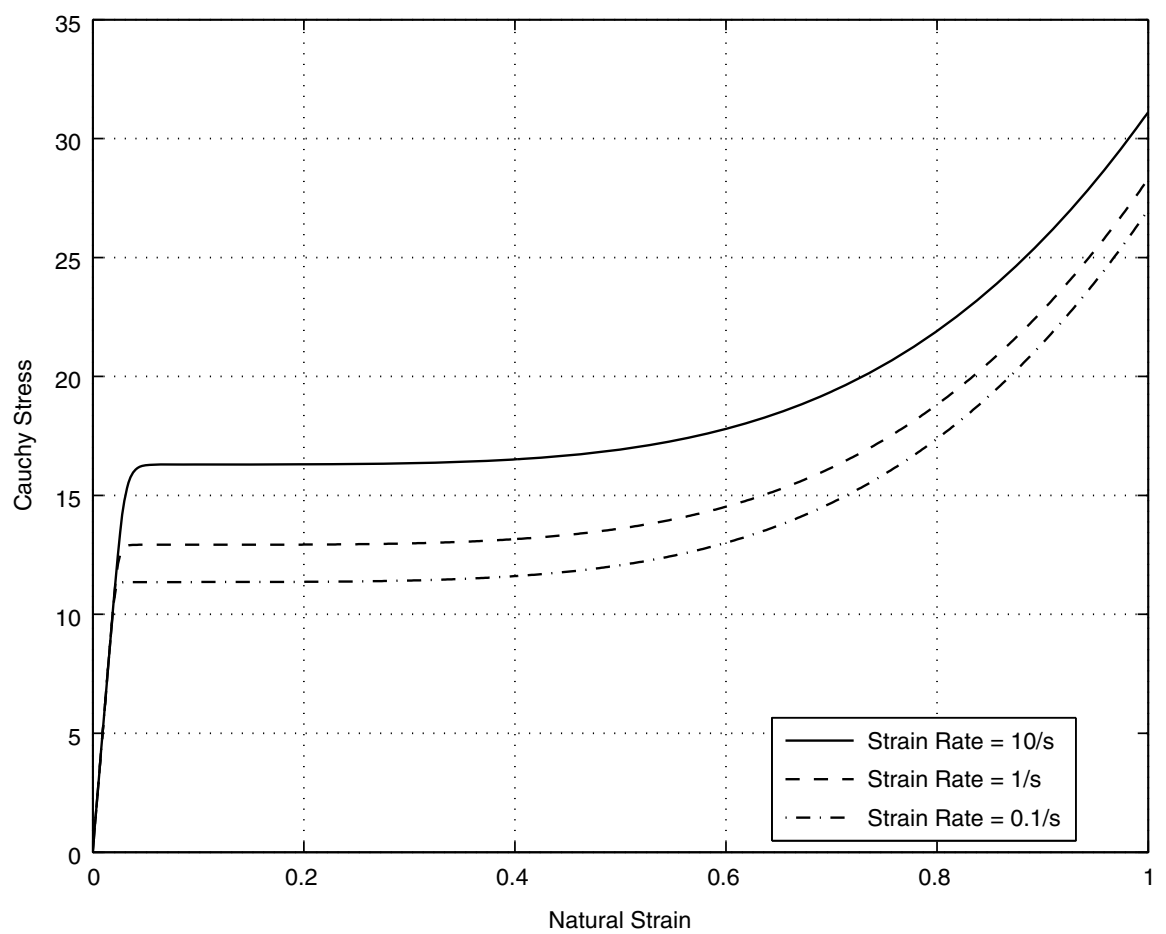

Fig. 14. Cauchy stress $\sigma_{x}$ versus natural strain. 


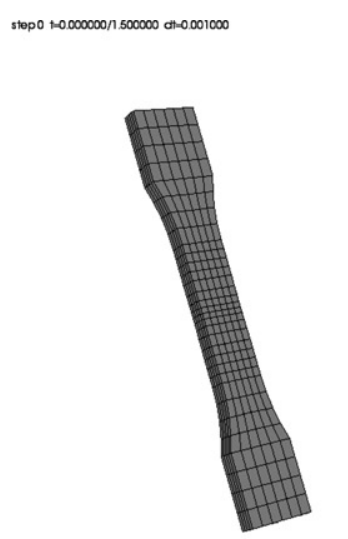

step $26+0.100000 / .500000$ atr-0.005000
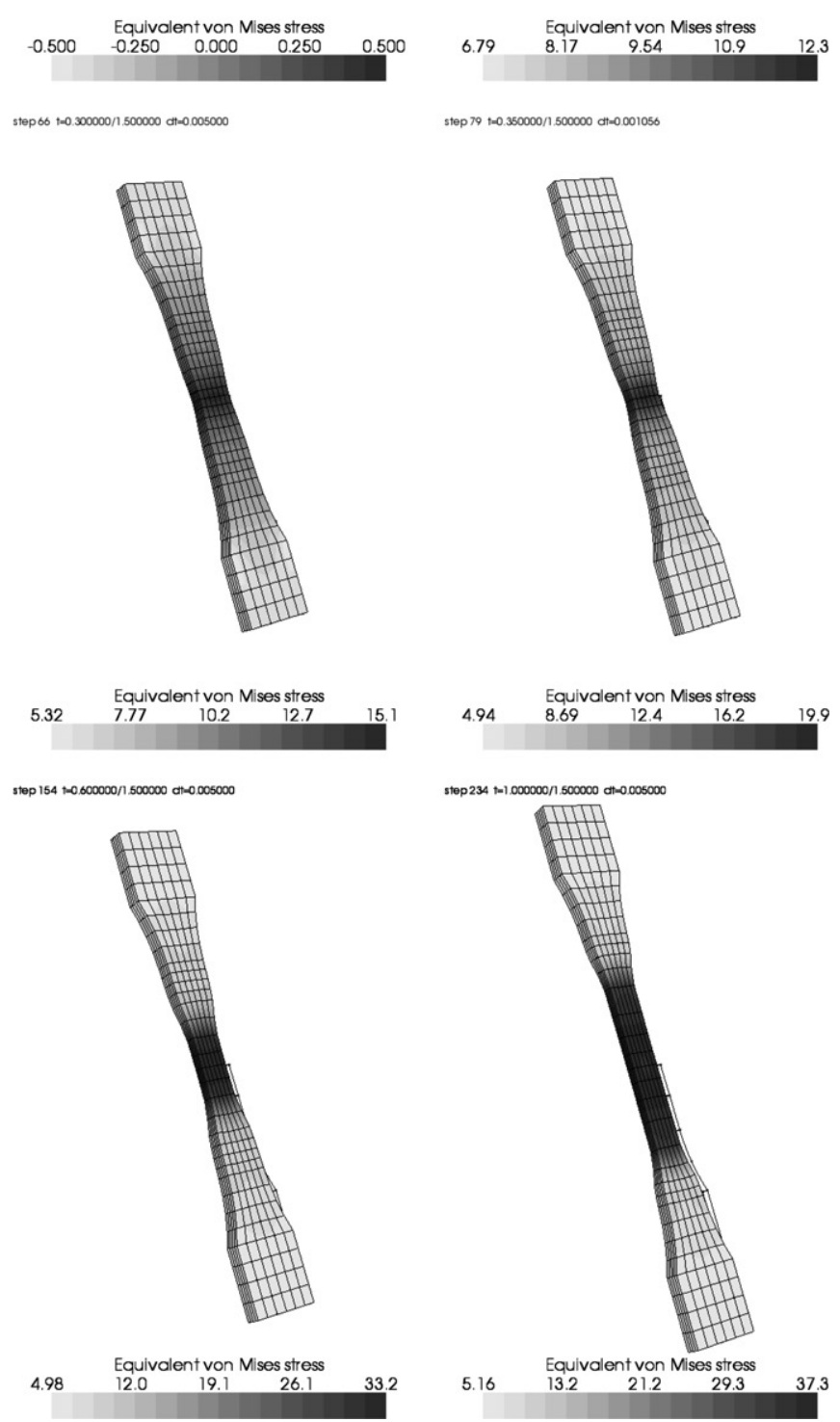

Fig. 15. Sequence of deformed configurations for displacement rate $30 \mathrm{~mm} / \mathrm{s}$.

shear stress $\sigma_{x y}$ equivalent stress $\sigma_{M}$ and equivalent strain $\Delta q$ is shown in Figs. 8-10 for the Hencky model and Figs. 11-13 for the Ogden model. As expected, a behavior similar to that of traction test is observed, with a clear nonlinear response for the elastic region in the Ogden case.

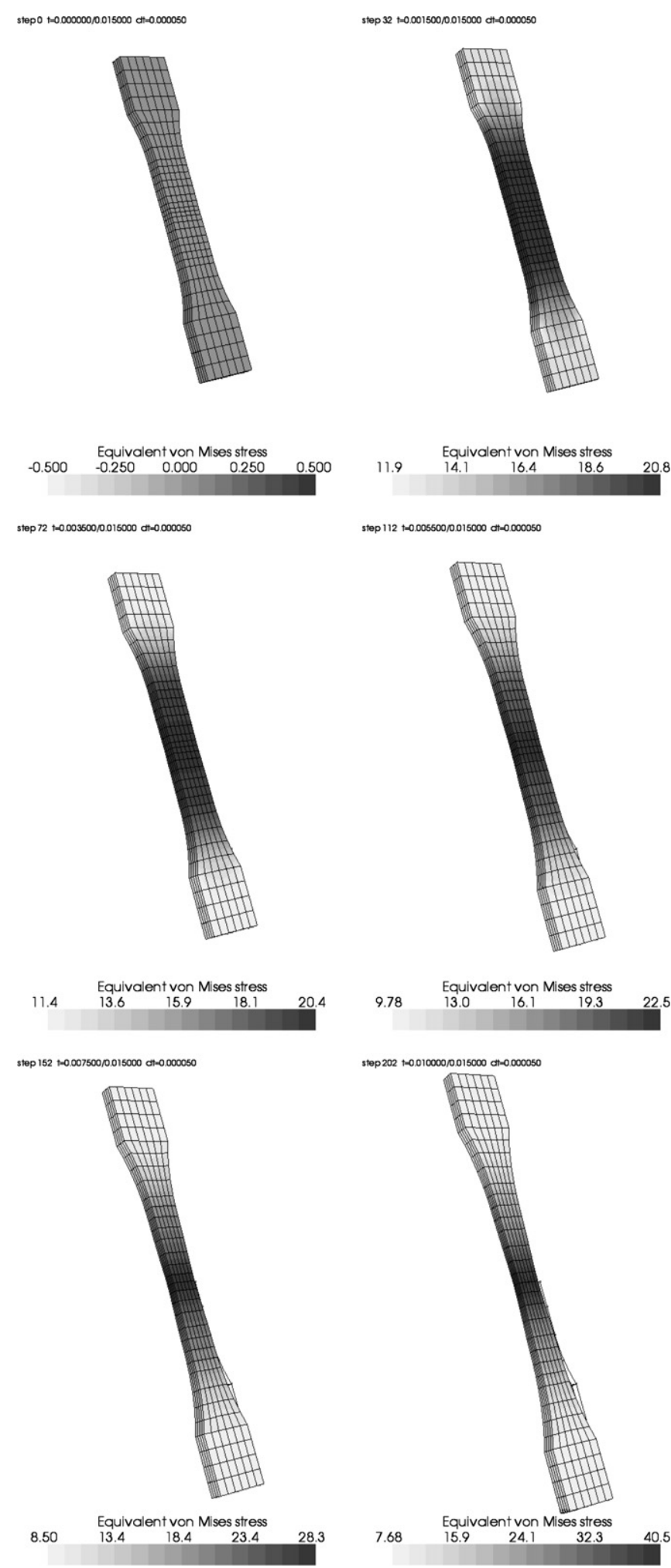

Fig. 16. Sequence of deformed configurations for displacement rate $300 \mathrm{~mm} / \mathrm{s}$.

\subsection{Normalized traction test}

The proposed formulation was implemented in the academic finite element code METAFOR [19]. The following example shows the simulation of an experimental traction test. The specimen geometry follows the ASTM D638-03 norm for thermoplastics 

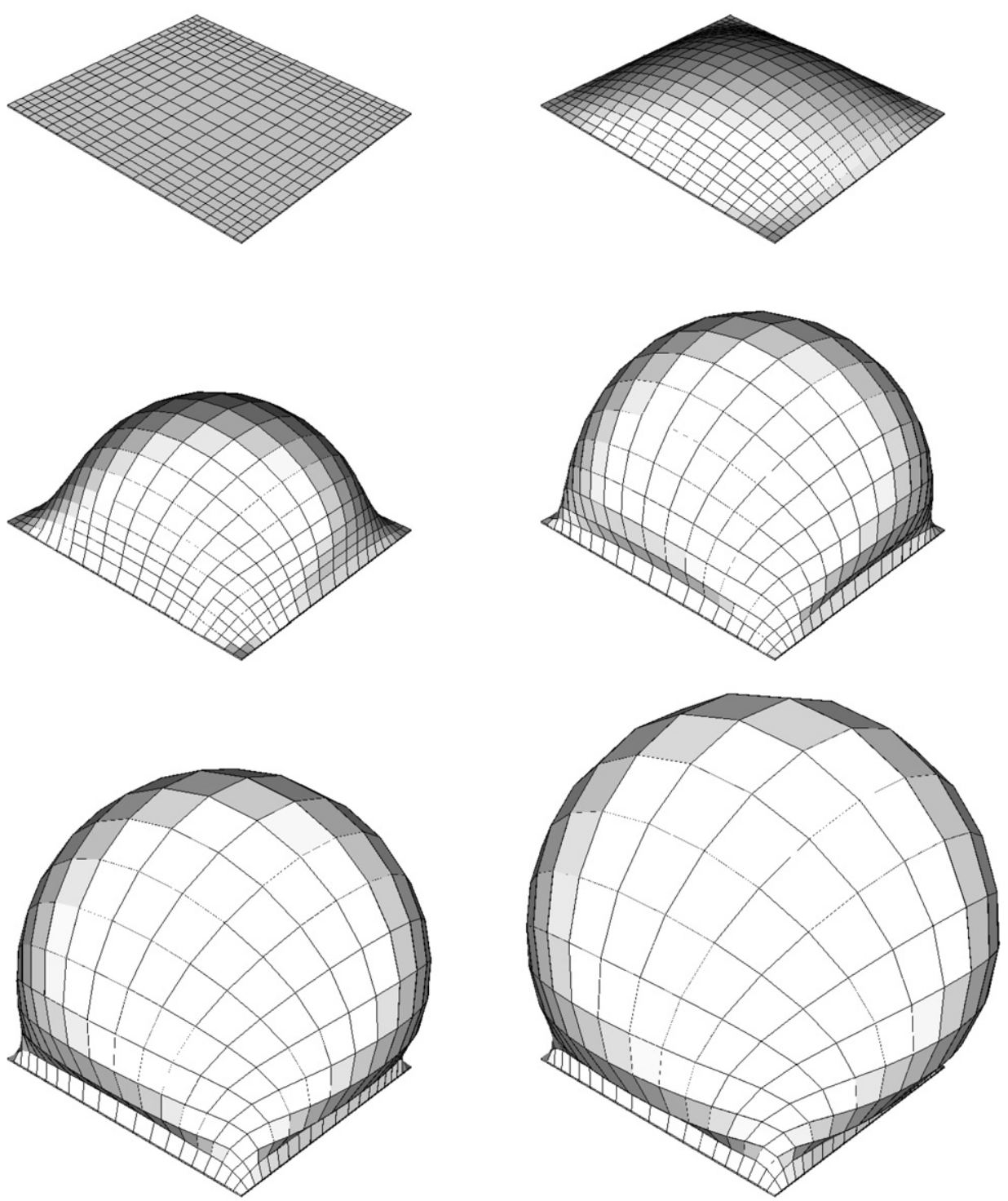

Fig. 17. Sequence of deformed configurations for pressure rate $0.1 \mathrm{~N} / \mathrm{mm}^{2} \mathrm{~s}$.

[20]. The material parameters are given in Table 2, values that provide the strain-stress curves found in Fig. 14. It is possible to see in these curves the usual behavior of some thermoplastics i.e. an elastic behavior followed by a large plateau of plastic deformation and a final hardening. The simulation of the traction test is performed by controlling the relative velocity of the specimen extremities, emulating a displacement-controlled real test. The specimen is elongated by $30 \mathrm{~mm}$ at rates of $30 \mathrm{~mm} / \mathrm{s}$ and $300 \mathrm{~mm} / \mathrm{s}$. The results of each case is shown in Figs. 15 and 16. The comparison between these two cases shows clear differences in the deformation shape due to differences in deformation rates of the material. In the slowest test, a clear necking nucleates ${ }^{5}$ and "grows" following the deformation of the specimen. Conversely, in the fastest test, the viscoplastic behavior inhibits a clear necking formation, producing a more homogeneous strain along the specimen, behavior usually found in these kind of materials.

\footnotetext{
${ }^{5}$ For both cases, the neck starts at the middle of the specimen due to a local small perturbation of its geometry.
}

\subsection{Inflated membrane}

This example shows the case of a membrane being inflated by a growing pressure. Used as a benchmark for rubbery materials, this example usually presents a snap-through behavior requiring an arc-length technique for a quasi-static solution. This snap-trough behavior is a result of an adequate combination of material behavior and geometrical characteristics. In the present case a squared viscoplastic membrane of $20 \mathrm{~mm}$ of length and thickness of $0.1 \mathrm{~mm}$ is submitted to a pressure rates of $1 \times 10^{-1} \mathrm{~N} / \mathrm{mm}^{2} \mathrm{~s}$, $1 \times 10^{-3} \mathrm{~N} / \mathrm{mm}^{2} \mathrm{~s}$ and $1 \times 10^{-5} \mathrm{~N} / \mathrm{mm}^{2} \mathrm{~s}$. The material is the same as the one used in the previous example.

Fig. 17 shows a sequence of deformed configurations of the membrane during loading. Fig. 18 show the diagram of classical von Mises stress at the bottom middle point. It is possible to see the dependence of stresses on pressure rate, as expected. Figs. 19 and 20 show in detail the behavior of stress and transversal displacement for a pressure up to $0.16 \mathrm{~N} / \mathrm{mm}^{2}$.

It is interesting to note that the expected snap-through phenomenon is not present here. This seems to happen due to the choice of a constitutive law with a low viscoplastic plateau. The hardening behavior of the material shown in Fig. 14 allows the 


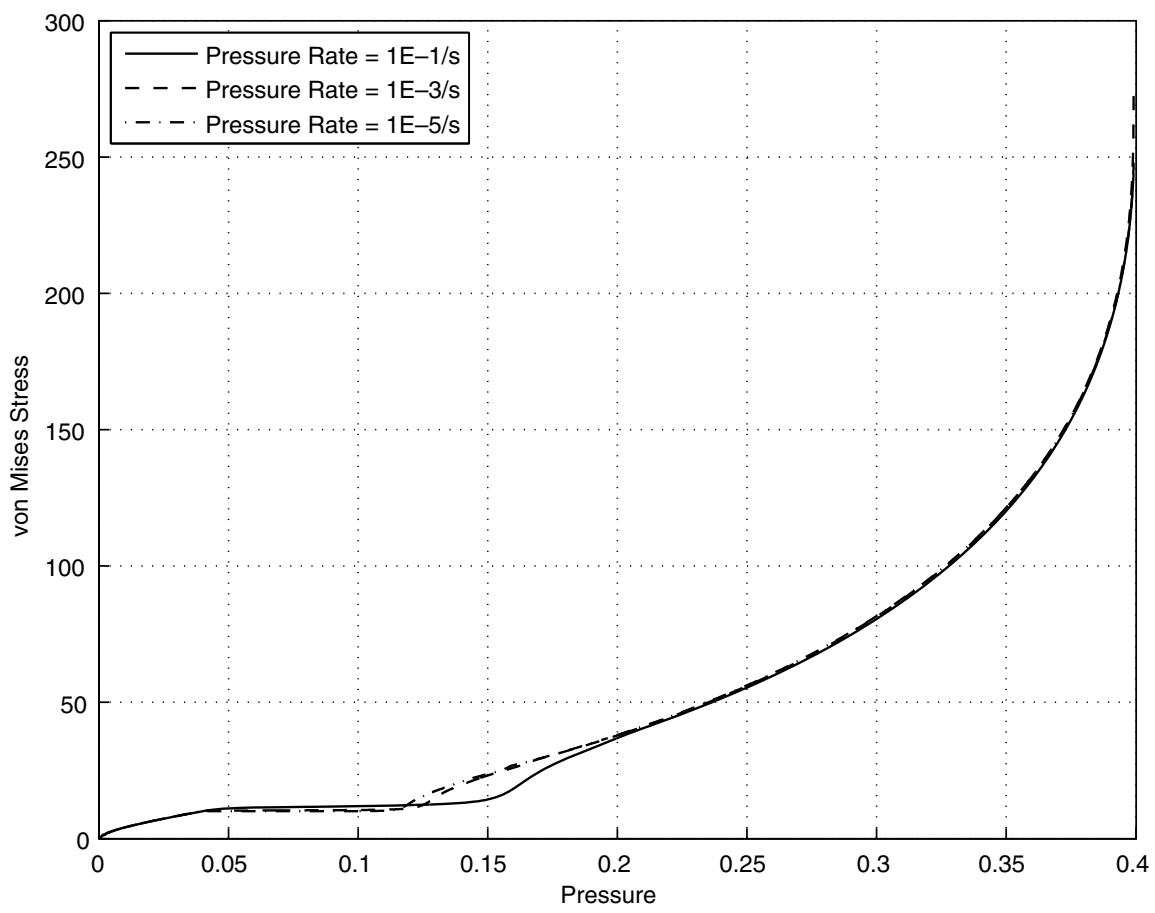

Fig. 18. von Mises stress versus pressure.

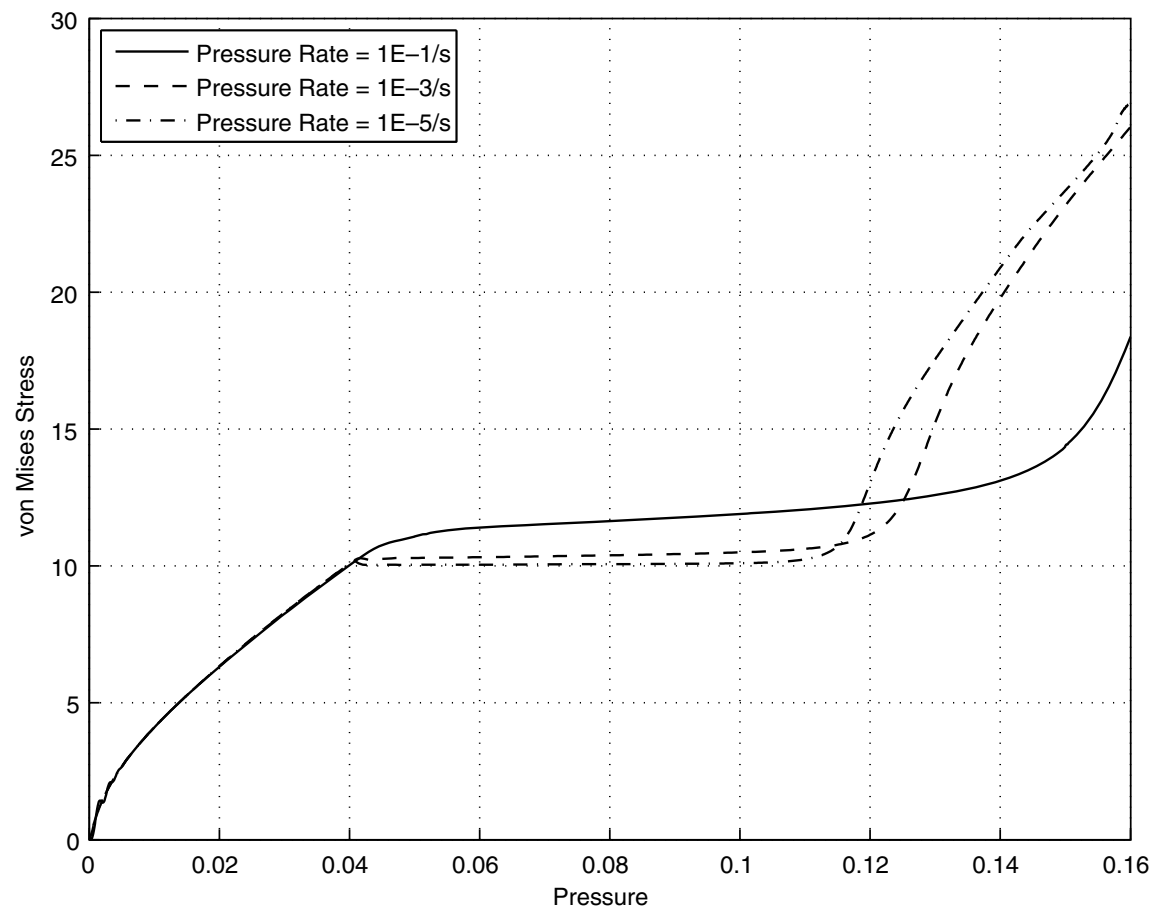

Fig. 19. von Mises stress versus pressure (detail).

membrane to reach high stress values. An instable configuration is found at pressure of approximately $0.4 \mathrm{~N} / \mathrm{mm}^{2}$ which is the upperlimit before failure. ${ }^{6}$ In the present case, a dynamic analysis with an artificial low density value was used instead of a quasi-static arclength technique.

${ }^{6}$ The material response shown in Fig. 14 is similar to that of some thermoplastics. The high stress values found at the end of present simulation are just numerical since a real thermoplastic would fail before that.

\section{Concluding remarks}

A general set of hyperelastic-viscoplastic material models was presented in this paper. Based on the variational approach of inelastic constitutive models [5], this work proposes as a main contribution, a spectral decomposition of viscoplastic strains which allows the use of generic hyperelastic and viscoplastic isotropic potential functions. Different material models may be obtained simply by changing the expression of these potentials and their derivatives. 


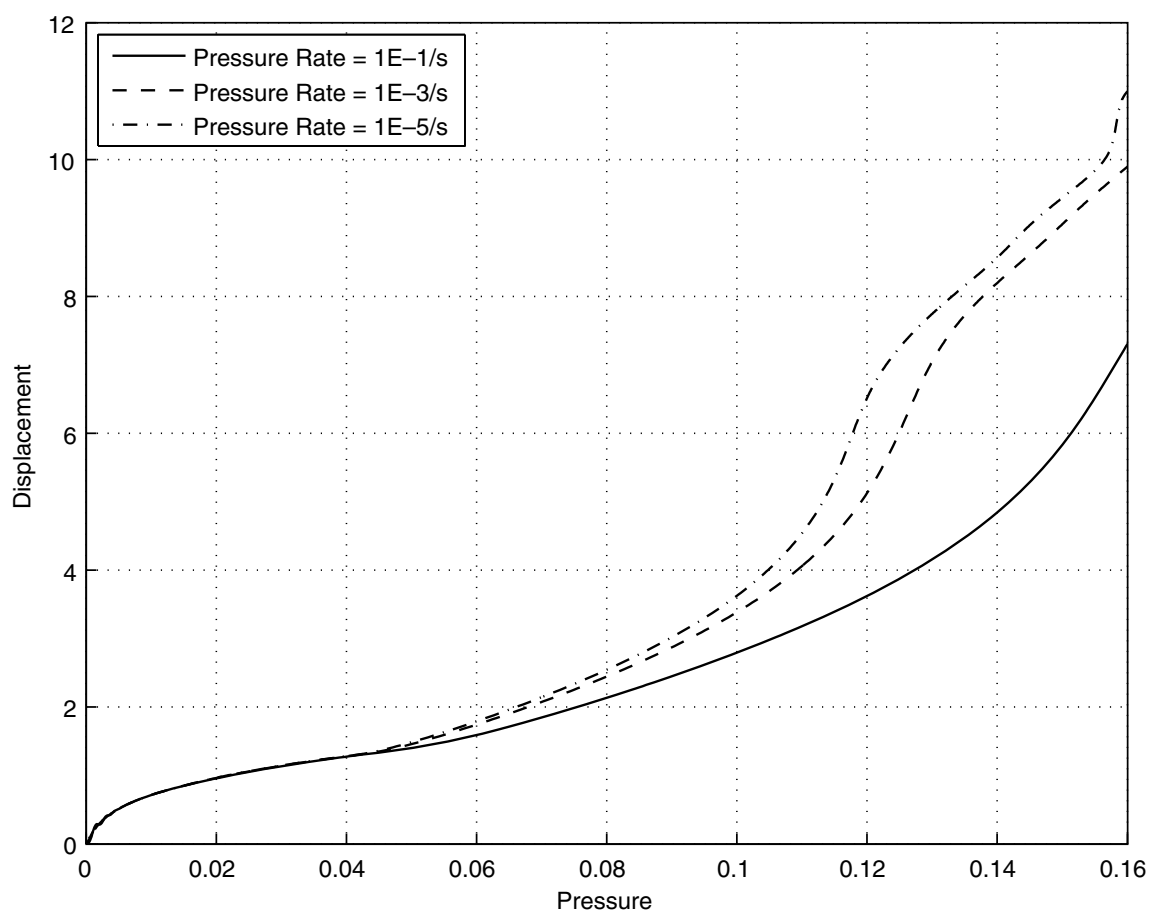

Fig. 20. Transversal displacement of central point versus pressure.

It is also shown that the incremental yield criterion comes out naturally from the minimization approach adopted here. In the isotropic case, this yield criterion amounts to evaluating the classical von Mises equivalent stress in terms of the Mandel stress tensor, in the intermediate configuration.

The stress and internal variables updates require (in the general case) the solution of a small set of nonlinear equations to determine the eigenvalues of viscoplastic increments. The solution of the nonlinear constitutive problem is based on the Newton method by using analytically invertible tangent matrices which provide computationally inexpensive material tensors for the global balance problem.

All these characteristics will allow us to combine this formulation with that already obtained for nonlinear viscoelastic behavior [11] in order to propose consistent models for polymeric materials subject to combined viscoelastic and viscoplastic strains, which is the subject of future works.

\section{Acknowledgements}

Eduardo Fancello thanks the Brazilian "Conselho Nacional de Desenvolvimento Científico e Tecnológico” who provided financial support for this research. Laurent Stainier thanks the Belgian "Fonds de la Recherche Scientifique - FNRS" for its support through his Research Associate position. We also thank the development team of METAFOR for the permission of use and modification of the code.

\section{Appendix}

\section{Minimization of $\boldsymbol{\Psi}$ with respect to $\mathbf{M}_{a}$}

The arguments that minimize the potential $\Psi\left(\Delta q, q_{i}, \mathbf{M}_{i}\right)$ must be such that $\Delta t \mathbf{D}^{p}=\Delta q \sum_{i=1}^{3} q_{i} \mathbf{M}_{i}$ is traceless and its decomposition is a sum of orthonormal symmetric tensors. This means that $q_{i} \in K_{Q}=\left\{p_{i} \in \mathbb{R}: \sum_{i=1}^{3} p_{i}=0 ; \quad \sum_{i=1}^{3} p_{i}^{2}=3 / 2\right\}$,

$\mathbf{M}_{i} \in K_{M}=\left\{\mathbf{M}_{i} \in \operatorname{Sym}: \mathbf{M}_{i} \cdot \mathbf{M}_{i}=1, \quad \mathbf{M}_{i} \cdot \mathbf{M}_{j}=0, \quad i \neq j\right\}$.

In order to enforce these constraints, the optimality condition is computed with the aid of a Lagrangian function that adds to the original potential all the considered equality constraints (the constraint on $\Delta q$, due to its simplicity is taken into account a posteriori):

$$
\begin{aligned}
\mathscr{L}\left(\Delta q, q_{i}, \mathbf{M}_{i}, \lambda_{j}, \lambda, \beta\right)= & \Psi\left(\Delta q, q_{i}, \mathbf{M}_{i}\right)+\lambda_{1}\left(\mathbf{M}_{a} \cdot \mathbf{M}_{a}-1\right)+\lambda_{2}\left(\mathbf{M}_{a} \cdot \mathbf{M}_{b}\right) \\
& +\lambda_{3}\left(\mathbf{M}_{a} \cdot \mathbf{M}_{c}\right)+\lambda_{4}\left(\mathbf{M}_{b} \cdot \mathbf{M}_{b}-1\right)+\lambda_{5}\left(\mathbf{M}_{b} \cdot \mathbf{M}_{c}\right) \\
& +\lambda_{6}\left(\mathbf{M}_{c} \cdot \mathbf{M}_{c}-1\right)+\lambda\left(q_{a}+q_{b}+q_{c}\right)+\beta\left(q_{a}^{2}+q_{b}^{2}\right. \\
& \left.+q_{c}^{2}-3 / 2\right) .
\end{aligned}
$$

Since $q_{i}$ do not depend on $\mathbf{M}_{i}$, the optimality condition with respect to the eigenprojection $\mathbf{M}_{a}$ is:

$$
\begin{aligned}
\frac{\partial \mathscr{L}}{\partial \mathbf{M}_{a}}[\delta \mathbf{M}]= & \frac{\partial \Psi}{\partial \mathbf{M}_{a}} \cdot \delta \mathbf{M}+2 \lambda_{1}\left(\mathbf{M}_{a} \cdot \delta \mathbf{M}\right)+\lambda_{2}\left(\mathbf{M}_{b} \cdot \delta \mathbf{M}\right) \\
& +\lambda_{3}\left(\mathbf{M}_{c} \cdot \delta \mathbf{M}\right)=0 \quad \forall \delta \mathbf{M} \in \mathrm{Sym} .
\end{aligned}
$$

Taking $\delta \mathbf{M}$ equal to $\mathbf{M}_{a}, \mathbf{M}_{b}, \mathbf{M}_{c}$ respectively, we obtain the relations

$$
\begin{aligned}
& 2 \lambda_{1}=\frac{\partial \Psi}{\partial \mathbf{M}_{a}} \cdot \mathbf{M}_{a}, \\
& \lambda_{2}=-\frac{\partial \Psi}{\partial \mathbf{M}_{a}} \cdot \mathbf{M}_{b}, \\
& \lambda_{3}=-\frac{\partial \Psi}{\partial \mathbf{M}_{a}} \cdot \mathbf{M}_{c} .
\end{aligned}
$$

Substituting them in (73),

$$
\begin{aligned}
& {\left[\frac{\partial \Psi}{\partial \mathbf{M}_{a}}-\left(\frac{\partial \Psi}{\partial \mathbf{M}_{a}} \cdot \mathbf{M}_{a}\right) \mathbf{M}_{a}-\left(\frac{\partial \Psi}{\partial \mathbf{M}_{a}} \cdot \mathbf{M}_{b}\right) \mathbf{M}_{b}\right.} \\
& \left.-\left(\frac{\partial \Psi}{\partial \mathbf{M}_{a}} \cdot \mathbf{M}_{c}\right) \mathbf{M}_{c}\right] \cdot \delta \mathbf{M}=0 \quad \forall \delta \mathbf{M} \in \text { Sym }
\end{aligned}
$$


The only term of $\Psi$ that depends on $\mathbf{M}_{i}$ is the elastic potential $\varphi^{e}\left(\epsilon_{i}^{e}\right)$ through the elastic tensor $\epsilon^{e}$, i.e,

$$
\begin{gathered}
\boldsymbol{\epsilon}^{e}=\sum_{i=1}^{3} \epsilon_{i}^{e} \mathbf{E}_{i}^{e}=\boldsymbol{\epsilon}^{p r}-\Delta t \mathbf{D}^{p}=\boldsymbol{\epsilon}^{p r}-\Delta q \sum_{i=1}^{3} q_{i} \mathbf{M}_{i} ; \quad \frac{\partial \epsilon_{a}^{e}}{\partial \boldsymbol{\epsilon}^{e}}=\mathbf{E}_{a}^{e}, \\
\frac{\partial \Psi}{\partial \mathbf{M}_{a}}=\frac{\partial \Delta \varphi^{e}}{\partial \mathbf{M}_{a}}=\sum_{i=1}^{3} \frac{\partial \Delta \varphi^{e}}{\partial \epsilon_{i}^{e}} \frac{\partial \epsilon_{i}^{e}}{\partial \boldsymbol{\epsilon}^{e}} \frac{\partial \boldsymbol{\epsilon}^{e}}{\partial \mathbf{M}_{a}}=-\left(\sum_{i=1}^{3} \frac{\partial \Delta \varphi^{e}}{\partial \epsilon_{i}^{e}} \mathbf{E}_{i}^{e}\right) \Delta q q_{a} .
\end{gathered}
$$

Substituting these result in (74), we have

$$
\begin{gathered}
{\left[\sum_{j=1}^{3} \frac{\partial \Delta \varphi^{e}}{\partial \epsilon_{i}^{e}} \mathbf{E}_{i}^{e}-\sum_{i=1}^{3} \frac{\partial \Delta \varphi^{e}}{\partial \epsilon_{i}^{e}}\left(\mathbf{E}_{i}^{e} \cdot \mathbf{M}_{a}^{v}\right) \mathbf{M}_{a}^{v}-\sum_{i=1}^{3} \frac{\partial \Delta \varphi^{e}}{\partial \epsilon_{i}^{e}}\left(\mathbf{E}_{i}^{e} \cdot \mathbf{M}_{b}^{v}\right) \mathbf{M}_{b}^{v}\right.} \\
\left.-\sum_{i=1}^{3} \frac{\partial \Delta \varphi^{e}}{\partial \epsilon_{i}^{e}}\left(\mathbf{E}_{i}^{e} \cdot \mathbf{M}_{c}^{v}\right) \mathbf{M}_{c}^{v}\right] \cdot \delta \mathbf{M}=0 \quad \forall \delta \mathbf{M} \in \text { Sym. }
\end{gathered}
$$

The last equation is satisfied if we take $\mathbf{E}_{i}^{e}=\mathbf{M}_{i}^{v}$.

Minimizing operations for $\Delta q, q_{i}$

Assuming that the material point performs a plastic increment $\Delta q>0$, the nonlinear system (35)-(38) must be solved. Using Newton method we find the roots $\mathbf{x}=\left\{q_{1}, q_{2}, q_{3}, \Delta q, \lambda, \beta\right\}$ using the recursive formula

$\mathbf{x}^{k+1}=\mathbf{x}^{k}-\mathbf{K}^{k-1} \mathbf{r}^{k}$,

where $k$ is the iteration number. In case that the potential $\varphi^{e}$ can be additively written as $\varphi^{e}\left(\epsilon_{i}^{e}\right)=\sum_{i=1}^{3} w_{i}^{e}\left(\epsilon_{i}^{e}\right)$, the material tangent matrix $\mathbf{K}$ takes the simplified form

$\mathbf{K}=\left[\begin{array}{cccccc}K_{11} & 0 & 0 & H_{1} & 1 & 2 q_{1} \\ 0 & K_{22} & 0 & H_{2} & 1 & 2 q_{2} \\ 0 & 0 & K_{33} & H_{3} & 1 & 2 q_{3} \\ H_{1} & H_{2} & H_{3} & H_{4} & 0 & 0 \\ 1 & 1 & 1 & 0 & 0 & 0 \\ 2 q_{1} & 2 q_{2} & 2 q_{3} & 0 & 0 & 0\end{array}\right]$,

$K_{i i}=\frac{\partial^{2} \varphi^{e}}{\partial \epsilon_{i}^{e} \partial \epsilon_{i}^{e}} \Delta q^{2}+2 \beta, \quad i=1,2,3$ (no index sum),

$H_{i}=\frac{\partial^{2} \Delta \varphi^{e}}{\partial \epsilon_{i}^{e} \partial \epsilon_{i}^{e}} q_{i} \Delta q-\frac{\partial \Delta \varphi^{e}}{\partial \epsilon_{i}^{e}}, \quad i=1,2,3$ (no index sum),

$H_{4}=\sum_{i=1}^{3} \frac{\partial^{2} \varphi^{e}}{\partial \epsilon_{i}^{e} \partial \epsilon_{i}^{e}} q_{i}^{2}+\frac{\partial^{2} \Delta \varphi^{p}}{\partial \Delta q^{2}}+\frac{1}{\Delta t} \frac{\partial^{2} \psi}{\partial \dot{q}^{2}}$.

Derivatives of $\varphi^{e}$ with respect to $\widehat{\mathbf{C}}_{n+1}$

In order to compute the derivative $\varphi^{e}$ we use the relation $\widehat{\mathbf{C}}^{p r}=\widehat{\mathbf{F}}^{p r^{\mathrm{T}}} \widehat{\mathbf{F}}^{p r}=\mathbf{F}_{n}^{p-\mathrm{T}} \widehat{\mathbf{C}}_{n+1} \mathbf{F}_{n}^{p-1}$

the derivative of $\varphi^{e}$ comes

$$
\begin{aligned}
\frac{\partial \varphi^{e}}{\partial \widehat{\mathbf{C}}_{n+1}}[\delta \mathbf{C}] & \left.=\frac{\partial \varphi^{e}}{\partial \widehat{\mathbf{C}}^{p r}} \cdot \frac{\partial \widehat{\mathbf{C}}^{p r}}{\partial \widehat{\mathbf{C}}_{n+1}}[\delta \mathbf{C}]=\frac{\partial \varphi^{e}}{\partial \widehat{\mathbf{C}}^{p r}} \cdot \mathbf{F}_{n}^{p-\mathrm{T}} \frac{\partial \widehat{\mathbf{C}}_{n+1}}{\partial \widehat{\mathbf{C}}_{n+1}}[\delta \mathbf{C}]\right) \mathbf{F}_{n}^{p-1} \\
& =\frac{\partial \varphi^{e}}{\partial \widehat{\mathbf{C}}^{p r}} \cdot \mathbf{F}_{n}^{p-\mathrm{T}} \frac{1}{2}\left(\delta \mathbf{C}+\delta \mathbf{C}^{\mathrm{T}}\right) \mathbf{F}_{n}^{p-1}=\mathbf{F}_{n}^{p-1} \frac{\partial \varphi^{e}}{\partial \widehat{\mathbf{C}}^{p r}} \mathbf{F}_{n}^{p-\mathrm{T}} \cdot \delta \mathbf{C}
\end{aligned}
$$

and then,

$\frac{\partial \varphi^{e}}{\partial \widehat{\mathbf{C}}_{n+1}}=\mathbf{F}_{n}^{p-1} \frac{\partial \varphi^{e}}{\partial \widehat{\mathbf{C}}^{p r}} \mathbf{F}_{n}^{p-\mathrm{T}}$,

where

$$
\frac{\partial \varphi^{e}}{\partial \widehat{\mathbf{C}}^{p r}}=\sum_{i=1}^{3} \frac{\partial \varphi^{e}}{\partial c_{i}^{p r}} \frac{\partial c_{i}^{p r}}{\partial \widehat{\mathbf{C}}_{n+1}^{p r}}=\sum_{j=1}^{3} \frac{\partial \varphi^{e}}{\partial c_{i}^{p r}} \mathbf{E}_{i}^{p r} .
$$

\section{Material tensors}

Considering $\widehat{\mathbf{C}}^{p r}=\left(\widehat{\mathbf{F}}^{p}\right)_{n}^{-\mathrm{T}} \widehat{\mathbf{C}}_{n+1}\left(\widehat{\mathbf{F}}^{p}\right)_{n}^{-1}$, calling $\mathbf{f}^{p n}=\left(\widehat{\mathbf{F}}^{p}\right)_{n}^{-1}$ and dropping index $n+1$, we have (in this particular expression we use Einstein's notation for summation on indices $p, q, m, t, u, v, r$, and $s)$ :

$$
\begin{aligned}
\frac{\mathrm{d}}{\mathrm{d} \widehat{\mathbf{C}}_{k l}}\left(\frac{\partial \varphi^{e}}{\partial \widehat{\mathbf{C}}_{i j}}\right) & =\frac{\mathrm{d}}{\mathrm{d} \widehat{\mathbf{C}}_{p q}^{p r}}\left(\frac{\partial \varphi^{e}}{\partial \widehat{\mathbf{C}}_{m t}^{p r}} \frac{\partial \widehat{\mathbf{C}}_{m t}^{p r}}{\partial \widehat{\mathbf{C}}_{i j}}\right) \frac{\partial \widehat{\mathbf{C}}_{p q}^{p r}}{\partial \widehat{\mathbf{C}}_{k l}}, \\
& =\frac{\mathrm{d}}{\mathrm{d} \widehat{\mathbf{C}}_{p q}^{p r}} \frac{\partial \varphi^{e}}{\partial \widehat{\mathbf{C}}_{m t}^{p r}} \frac{\partial\left(\mathbf{f}_{p u}^{p n^{\mathrm{T}}} \widehat{\mathbf{C}}_{u v} \mathbf{f}_{v q}^{p n}\right)}{\partial \widehat{\mathbf{C}}_{k l}} \frac{\partial\left(\mathbf{f}_{m r}^{p n^{\mathrm{T}}} \widehat{\mathbf{C}}_{r s} \mathbf{f}_{s t}^{p n}\right)}{\partial \widehat{\mathbf{C}}_{i j}}, \\
& =\frac{\mathrm{d}}{\mathrm{d} \widehat{\mathbf{C}}_{p q}^{p r}} \frac{\partial \varphi^{e}}{\partial \widehat{\mathbf{C}}_{m t}^{p r}} \mathbf{f}_{p u}^{p n^{\mathrm{T}}} \mathscr{I}_{u v k l} \mathbf{f}_{v q}^{p n} \mathbf{f}_{m r}^{p n^{\mathrm{T}}} \mathscr{I}_{r s i j} \mathbf{f}_{s t}^{p n}, \\
& =\mathbf{f}_{r m}^{p n} \mathbf{f}_{s t}^{p n} \frac{\mathrm{d}}{\mathrm{d} \widehat{\mathbf{C}}_{p q}^{p r}} \frac{\partial \varphi^{e}}{\partial \widehat{\mathbf{C}}_{m t}^{p r}} \mathbf{f}_{u p}^{p n} \mathbf{f}_{v q}^{p n} \mathscr{I}_{u v k l} \mathscr{I}_{r s i j}, \\
& =\mathbf{f}_{i m}^{p n} \mathbf{f}_{j t}^{p n} \frac{\mathrm{d}}{\mathrm{d} \widehat{\mathbf{C}}_{p q}^{p r}} \frac{\partial \varphi^{e}}{\partial \widehat{\mathbf{C}}_{m t}^{p r}} \mathbf{f}_{k p}^{p n} \mathbf{f}_{l q}^{p n}=\mathscr{C}_{j i k l}^{\varphi^{e}}, \\
\mathscr{C}_{i j k l}^{\varphi^{e}} & =\mathscr{C}_{k l i j}^{\varphi^{e}}, \quad \mathscr{C}_{i j k l}^{\varphi^{e}}=\mathscr{C}_{j i k l}^{\varphi^{e}} .
\end{aligned}
$$

The total derivative of $\varphi^{e}$ with respect to $\epsilon_{j}^{p r}$ comes from the derivative of the nonlinear optimality conditions. Assuming for simplicity purposes $\varphi^{e}\left(\epsilon_{i}^{e}\right)=\sum_{i=1}^{3} w_{i}^{e}\left(\epsilon_{i}^{e}\right)$ and considering that

$\epsilon_{i}^{e}=\epsilon_{i}^{p r}-\Delta q q_{i} \Rightarrow \frac{\mathrm{d} \epsilon_{i}^{e}}{\mathrm{~d} \epsilon_{j}^{p r}}=\delta_{i j}-\frac{\mathrm{d} \Delta q}{\mathrm{~d} \epsilon_{j}^{p r}} q_{i}-\Delta q \frac{\mathrm{d} q_{i}}{\mathrm{~d} \epsilon_{j}^{p r}}$

we have

$$
\begin{aligned}
& \frac{\mathrm{d} r_{i}}{\mathrm{~d} \epsilon_{j}^{p r}}=-\frac{\partial^{2} \Delta \varphi^{e}}{\partial \epsilon_{i}^{e} \partial \epsilon_{i}^{e}} \frac{\mathrm{d} \epsilon_{i}^{e}}{\mathrm{~d} \epsilon_{j}^{p r}} \Delta q-\frac{\partial \Delta \varphi^{e}}{\partial \epsilon_{i}^{e}} \frac{\mathrm{d} \Delta q}{\mathrm{~d} \epsilon_{j}^{p r}}+\frac{\mathrm{d} \lambda}{\mathrm{d} \epsilon_{j}^{p r}}+2 \beta \frac{\mathrm{d} q_{i}}{\mathrm{~d} \epsilon_{j}^{p r}}+2 q_{i} \frac{\mathrm{d} \beta}{\mathrm{d} \epsilon_{j}^{p r}}=0 \\
& \left.=-\frac{\partial^{2} \Delta \varphi^{e}}{\partial \epsilon_{i}^{e} \partial \epsilon_{i}^{e}} \quad \delta_{i j}-\frac{\mathrm{d} \Delta q}{\mathrm{~d} \epsilon_{j}^{p r}} q_{i}-\Delta q \frac{\mathrm{d} q_{i}}{\mathrm{~d} \epsilon_{j}^{p r}}\right) \Delta q \\
& -\frac{\partial \Delta \varphi^{e}}{\partial \epsilon_{i}^{e}} \frac{\mathrm{d} \Delta q}{\mathrm{~d} \epsilon_{j}^{p r}}+\frac{\mathrm{d} \lambda}{\mathrm{d} \epsilon_{j}^{p r}}+2 \beta \frac{\mathrm{d} q_{i}}{\mathrm{~d} \epsilon_{j}^{p r}}+2 q_{i} \frac{\mathrm{d} \beta}{\mathrm{d} \epsilon_{j}^{p r}}=0 \\
& =\left(\varphi_{, i i}^{e} \Delta q^{2}+2 \beta\right) \frac{\mathrm{d} q_{i}}{\mathrm{~d} \epsilon_{j}^{p r}}+\left(\varphi_{, i i}^{e} q_{i} \Delta q-\varphi_{, i}^{e}\right) \frac{\mathrm{d} \Delta q}{\mathrm{~d} \epsilon_{j}^{p r}} \\
& +\frac{\mathrm{d} \lambda}{\mathrm{d} \epsilon_{j}^{p r}}+2 q_{i} \frac{\mathrm{d} \beta}{\mathrm{d} \epsilon_{j}^{p r}}-\varphi_{, i i}^{e} \delta_{i j} \Delta q=0 \\
& \Rightarrow K_{i i} \frac{\mathrm{d} q_{i}}{\mathrm{~d} \epsilon_{j}^{p r}}+H_{i} \frac{\mathrm{d} \Delta q}{\mathrm{~d} \epsilon_{j}^{p r}}+\frac{\mathrm{d} \lambda}{\mathrm{d} \epsilon_{j}^{p r}}+2 q_{i} \frac{\mathrm{d} \beta}{\mathrm{d} \epsilon_{j}^{p r}}=\varphi_{, i i}^{e} \delta_{i j} \Delta q, \\
& i, j=1,2,3 \text { (no index sum), } \\
& \left.\frac{\mathrm{d} r_{4}}{\mathrm{~d} \epsilon_{j}^{p r}}=-\sum_{i=1}^{3} \varphi_{, i i}^{e} q_{i} \frac{\mathrm{d} \epsilon_{i}^{e}}{\mathrm{~d} \epsilon_{j}^{p r}}-\sum_{i=1}^{3} \varphi_{, i}^{e} \frac{\mathrm{d} q_{i}}{\mathrm{~d} \epsilon_{j}^{p r}}+\frac{\partial^{2} \Delta \varphi^{p}}{\partial \Delta q^{2}}+\frac{1}{\Delta t} \frac{\partial^{2} \psi}{\partial \dot{q}^{2}}\right) \frac{\mathrm{d} \Delta q}{\mathrm{~d} \epsilon_{j}^{p r}}=0 \\
& =\sum_{i=1}^{3}\left(\varphi_{, i i}^{e} q_{i} \Delta q-\varphi_{, i}^{e}\right) \frac{\mathrm{d} q_{i}}{\mathrm{~d} \epsilon_{j}^{p r}} \\
& \left.+\sum_{i=1}^{3} \varphi_{, i i}^{e} q_{i}^{2}+\frac{\partial^{2} \Delta \varphi^{p}}{\partial \Delta q^{2}}+\frac{1}{\Delta t} \frac{\partial^{2} \psi}{\partial \dot{q}^{2}}\right) \frac{\mathrm{d} \Delta q}{\mathrm{~d} \epsilon_{j}^{p r}}-\varphi_{, j j}^{e} q_{j}=0 \\
& \Rightarrow \sum_{i=1}^{3} H_{i} \frac{\mathrm{d} q_{i}}{\mathrm{~d} \epsilon_{j}^{p r}}+H_{4} \frac{\mathrm{d} \Delta q}{\mathrm{~d} \epsilon_{j}^{p r}}=\varphi_{. j j}^{e} q_{j}, \\
& i, j=1,2,3 \text { (no index sum), } \\
& \frac{\mathrm{d} r_{5}}{\mathrm{~d} \epsilon_{j}^{p r}}=\sum_{i=1}^{3} \frac{\mathrm{d} q_{i}}{\mathrm{~d} \epsilon_{j}^{p r}}=0 \\
& \frac{\mathrm{d} r_{6}}{\mathrm{~d} \epsilon_{j}^{p r}}=\sum_{i=1}^{3} 2 q_{i} \frac{\mathrm{d} q_{i}}{\mathrm{~d} \epsilon_{j}^{p r}}=0 .
\end{aligned}
$$

Or, in a compact notation,

$\mathbf{K} \mathbf{x}_{j}=Z \mathbf{y}_{j}$, 
$\mathbf{x}_{j}=\left[\frac{\mathrm{d} q_{1}^{e}}{\mathrm{~d} \epsilon_{j}^{p r}} \frac{\mathrm{d} q_{2}^{e}}{\mathrm{~d} \epsilon_{j}^{p r}} \frac{\mathrm{d} q_{3}^{e}}{\mathrm{~d} \epsilon_{j}^{p r}} \frac{\mathrm{d} \Delta q}{\mathrm{~d} \epsilon_{j}^{p r}} \frac{\mathrm{d} \lambda}{\mathrm{d} \epsilon_{j}^{p r}} \frac{\mathrm{d} \beta}{\mathrm{d} \epsilon_{j}^{p r}}\right]^{\mathrm{T}}$,

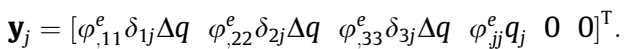

Matrix $\mathbf{K}$ is the converged tangent matrix (79). The solution of the system (90) provides the vector $\mathbf{x}_{j}$ that, together with (85) provides $\frac{\mathrm{d} \epsilon_{1}^{e}}{\mathrm{~d} e^{p l}}$. Further simplifications may be done according to the chosen potentials.

\section{Hencky-based model}

The residual for Hencky potentials take the form

$r_{i}=-2 \mu^{e}\left(\epsilon_{i}^{p r}-\Delta q q_{i}\right) \Delta q+\lambda+2 \beta q_{i}=0, \quad i=1,2,3$,

$r_{4}=-\sum_{i=1}^{3} 2 \mu^{e}\left(\epsilon_{i}^{p r}-\Delta q q_{i}\right) q_{i}+\Sigma_{0}+h q_{n+1}+Y_{0}=0$,

$r_{5}=\sum_{i=1}^{3} q_{i}=0, \quad r_{6}=\sum_{i=1}^{3} q_{i}^{2}=3 / 2$

Summing up all three equations (93) and considering (95) we have $\lambda=0$. From (94) and (95) we then obtain the compact expression

$a \Delta q+b=2 \mu^{e} \sum_{i=1}^{3} \epsilon_{i}^{p r} q_{i}$,

where

$a=3 \mu^{e}+H, \quad b=\Sigma_{0}+H q_{n}+Y_{0}$.

Summing up all three equations (93) multiplied by $q_{i}$, and subtracting (94) multiplied by $\Delta q$ we have

$\beta=\frac{1}{3} \Delta q\left(\Sigma_{0}+H q_{n}+H \Delta q+Y_{0}\right)$,

which is substituted in (93) to obtain

$q_{i}=\frac{3 \mu^{e} \epsilon_{i}^{p r}}{a \Delta q+b}$

Substituting (98) in (96) and noting

$s_{i}^{p r}=2 \mu^{e} \epsilon_{i}^{p r}, \quad\left\|\mathbf{s}^{p r}\right\|^{2}=\sum_{j=1}^{3}\left(s_{j}^{p r}\right)^{2}$,

we arrive, after simple manipulations, to the classic expression for the equivalent plastic increment
$\Delta q=\frac{\sqrt{\frac{3}{2}}\left\|\mathbf{s}^{p r}\right\|-\left(Y_{0}+\Sigma_{0}+h q_{n}\right)}{3 \mu^{e}+h}$.

\section{References}

[1] P. Mialon, Eléments d'analyse et de résolution numérique des relations de l'élasto-plasticité, EDF Bull. direction Etud. rech., Série C, Math., Inform. 3 (1986) 57-89.

[2] C. Comi, U. Perego, A unified approach for variationally consistent finite elements in elastoplasticity, Comput. Methods Appl. Mech. Engrg. 121 (1995) 323-344.

[3] J. Simo, T. Hughes, Computational Inelasticity, Springer, 1998.

[4] B. Halphen, Q.S. Nguyen, Sur les matériaux standard généralisés, J Mécanique 14 (1975) 39-63.

[5] M. Ortiz, L. Stainier, The variational formulation of viscoplastic constitutive updates, Comput. Methods Appl. Mech. Engrg. 171 (1999) 419-444.

[6] R. Radovitzky, M. Ortiz, Error estimation and adaptive meshing in strongly nonlinear dynamic problems, Comput. Methods Appl. Mech. Engrg. 172 (1999) 203-240.

[7] Q. Yang, L. Stainier, M. Ortiz, A variational formulation of the coupled thermomechanical boundary-value problem for general dissipative solids, J. Mech. Phys. Solids 74 (2) (2006) 401-424.

[8] J. Mosler, M. Ortiz, Variational $h$-adaption in finite deformation elasticity and plasticity, Int. J. Numer. Methods Engrg. 72 (2007) 505-523.

[9] M. Ortiz, A. Pandolfi, A variational Cam-clay theory of plasticity, Comput. Methods Appl. Mech. Engrg. 193 (2004) 2645-2666.

[10] K. Weinberg, A. Mota, M. Ortiz, A variational constitutive model for porous metal plasticity, Comput. Mech. 37 (2006) 142-152.

[11] E. Fancello, J.-P. Ponthot, L. Stainier, A variational formulation of constitutive models and updates in nonlinear finite viscoelasticity, Int. J. Numer. Methods Engrg. 65 (2006) 1831-1864.

[12] M. Wilkins, Calculation of elastic-plastic flow, in: B. Adler (Ed.), Methods of Computational Physics, vol. 3, Academic Press, New York, 1964.

[13] A.L. Eterovic, K.J. Bathe, A hyperelastic based large strain elasto-plastic constitutive formulation with combined isotropic-kinematic hardening using logarithmic stress and strain measures, Int. J. Numer. Methods Engrg. 30 (1990) 1099-1114.

[14] A. Cuitino, M. Ortiz, A material-independent method for extending stress update algorithms for small-strain plasticity to finite plasticity with multiplicative kinematics, Engrg. Comput. 9 (1992) 437-451.

[15] M.A. Crisfield, Non-linear Finite Element Analysis of Solids and Structures, John Wiley and Sons, England, 2001.

[16] E.S. Neto, D. Peric, D.R.J. Owen, Comput. Methods Plasticity, to be published.

[17] G. Weber, L. Anand, Finite deformations constitutive equations and a time integration procedure for isotropic hyperelastic-viscoplastic solids, Comput. Methods Appl. Mech. Engrg. 79 (1990) 173-202.

[18] G. Holzapfel, Nonlinear Solid Mechanics, Wiley, Chichester, England, 2000.

[19] Mécanique Numérique Non Linéaire - Département Aérospatiale \& Mécanique, Université de Liège, Belgium, METAFOR - Software for thermomechanical analysis of structures in large strain regime, <http://www.ltasmnl.ulg.ac.be>.

[20] ASTM Standard D638-03: Standard Test Method for Tensile Properties of Plastics, 2003. 